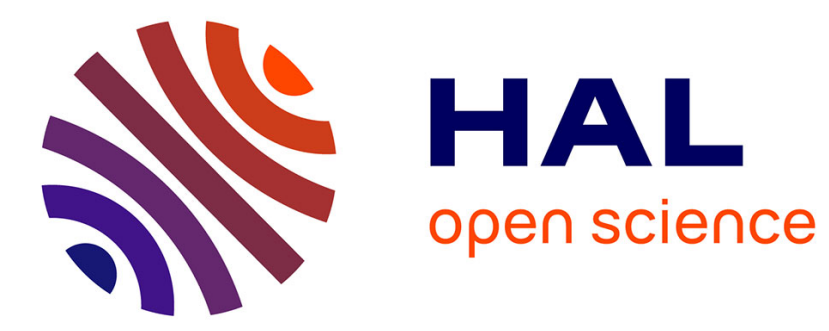

\title{
DISCONTINUOUS-GALERKIN DISCRETIZATION OF A NEW CLASS OF GREEN-NAGHDI EQUATIONS
}

\author{
Arnaud Duran, Fabien Marche
}

\section{- To cite this version:}

Arnaud Duran, Fabien Marche. DISCONTINUOUS-GALERKIN DISCRETIZATION OF A NEW CLASS OF GREEN-NAGHDI EQUATIONS. 2014. hal-00980826v1

\section{HAL Id: hal-00980826 \\ https://hal.science/hal-00980826v1}

Preprint submitted on 25 Apr 2014 (v1), last revised 10 Oct 2014 (v2)

HAL is a multi-disciplinary open access archive for the deposit and dissemination of scientific research documents, whether they are published or not. The documents may come from teaching and research institutions in France or abroad, or from public or private research centers.
L'archive ouverte pluridisciplinaire HAL, est destinée au dépôt et à la diffusion de documents scientifiques de niveau recherche, publiés ou non, émanant des établissements d'enseignement et de recherche français ou étrangers, des laboratoires publics ou privés. 


\title{
DISCONTINUOUS-GALERKIN DISCRETIZATION OF A NEW CLASS OF GREEN-NAGHDI EQUATIONS
}

\author{
A.DURAN ${ }^{1,2}$ AND F.MARCHE ${ }^{1,2}$
}

\begin{abstract}
We describe in this work a discontinuous-Galerkin Finite-Element method to approximate the solutions of a new family of $1 \mathrm{~d}$ Green-Naghdi models. These new models are shown to be more computationally efficient, while being asymptotically equivalent to the initial formulation with regard to the shallowness parameter. Using the free surface instead of the water height as a conservative variable, the models are recasted under a pre-balanced formulation and discretized using an expansion basis of arbitrary order. Independently from the polynomial degree in the approximation space, the preservation of the motionless steady-states is automatically ensured, and the water height positivity is enforced. A simple numerical procedure devoted to handle broken waves is also described. The validity of the resulting model is assessed through extensive numerical validations.
\end{abstract}

\section{INTRODUCTION}

Depth-averaged equations are widely used in coastal engineering for the simulation of nonlinear waves propagation and transformations in nearshore areas. The full description of surface water waves in an incompressible, homogeneous, inviscid fluid, is provided by the free surface Euler (or water waves) equations but this problem remains mathematically and numerically challenging. As a consequence, the use of depth averaged equations helps to reduce the three-dimensional problem to a two-dimensional problem, while keeping a good level of accuracy in many configurations.

Many Boussinesq-like models are used nowadays and a detailed review can be found in [42] and the recent monograph [41]. Denoting by $\lambda$ the typical horizontal scale of the flow and $h_{0}$ the typical depth, the shallow water regime usually corresponds to the configuration where $\mu:=\frac{h_{0}^{2}}{\lambda^{2}} \ll 1$. If approximations of order $O\left(\mu^{2}\right)$ of the free surface Euler equations are furnished by the Boussinesq-type (BT equations in the following) equations, see $[52,54,57]$ for instance, an additional smallness amplitude assumption on the typical wave amplitude $a$ is classically performed: $\varepsilon:=\frac{a}{h_{0}}=O(\mu)$. This assumption often appears as too restrictive for many applications in coastal oceanography. Removing the small amplitude assumption while still keeping all the $O(\mu)$ terms, we obtain the so-called Green-Naghdi equations (GN equations in the following) [34], also referred to as Serre equations [62] or fully non-linear Boussinesq equations [76].

A large number of numerical methods have been developed in the past few years for the BT equations. Let us mention for instance some Finite-Difference (FDM in the following) approaches [49, 54,65, 75], Finite-Element methods (FEM in the following) [46, 59,66,73], Finite-Volume discretizations (FVM in the following) for $1 \mathrm{~d}$ equations [21], hybrid FDM / FVM [24, 25, 55, 64,69], or even a purely 2d FVM discretization on unstructured

\footnotetext{
${ }^{1}$ Institut de Mathématiques et de Modélisation de Montpellier (I3M), Université Montpellier 2, CC 051, 34090 Montpellier, France

2 INRIA, team LEMON, 95 rue de la Galéra, 34090 Montpellier, France 
meshes [37], allowing for mesh refinement and flexibility for large scale simulations.

As far as flexibility is concerned, the use of discontinuous-Galerkin methods (dG methods in the following) would appear as a natural choice. Indeed, this class of method provides several appealing features, like compact discretization stencils and hp-adaptivity, flexibility with a natural handling of unstructured meshes, easy parallel computation and local conservation properties in the approximation of conservation laws. A general review of $\mathrm{dG}$ methods for convection dominated problems is performed in [14]. Concerning the approximation of more general problems, involving higher-order derivatives, several methods and important developments have been proposed in recent years, following [4] on Navier-Stokes equations and [15] on convection-diffusion systems. A recent review is performed in [80] and a unified analysis can be found in [3], and [26,27], respectively for elliptic problems and both $1^{\text {st }}$ and $2^{\text {nd }}$ order problems in the framework of Friedrichs' systems.

The application of dG methods to the Saint-Venant equations (also called Nonlinear Shallow Water equations, NSW in the following) has recently lead to several improvements, see for instance $[2,28,78,79]$ and the recent review [19]. However, dG methods for BT equations have been under-investigated. In [29], a $h p /$ spectral element model is introduced for the $1 \mathrm{~d}$ enhanced equations of Nwogu [54], while the $2 \mathrm{~d}$ equations of Peregrine [57] are studied in [30], in the flat bottom case, relying on a scalar reformulation that allowed some computational savings. This formulation is further investigated in [31], accounting for variable depth, and in [32] with the study of the enhanced equations of Madsen and Sorensen [51]. In [22,23], an arbitrary order nodal dG-FEM method is developed for the set of highly-dispersive BT equations introduced in [50], respectively in $1 \mathrm{~d}$ and $2 \mathrm{~d}$ on unstructured meshes. These equations have a larger range of validity and can theoretically model fully nonlinear waves transformation, but they are also more complex, introducing a dependence in the vertical velocity, and consequently additional degrees of freedoms in the discrete approximations. As stated by the authors themselves, the bottleneck of their approach lies in the need of reconstruction and resolution of the large associated linear systems: this process can rapidly become a drawback for large-scale simulations.

Surprisingly, the GN equations have received far less attention. In the 1d framework and formulated in terms of free-surface elevation above the still water depth $\zeta$ and horizontal velocity $u$, these equations read as follows (see $[1,7]$ for the derivation of this particular formulation):

$$
\left\{\begin{array}{l}
\partial_{t} \zeta+\partial_{x}(h u)=0 \\
{[1+\alpha \mathbb{T}[z, h]]\left(\partial_{t}(h u)+\partial_{x}\left(h u^{2}\right)+\frac{\alpha-1}{\alpha} g h \partial_{x} \zeta\right)+\frac{1}{\alpha} g h \partial_{x} \zeta+h \mathcal{Q}_{1}(u)=0}
\end{array}\right.
$$

with the differential operators

$$
\mathcal{Q}_{1}(u)=2 h \partial_{x} h\left(\partial_{x} u\right)^{2}+\frac{4}{3} h^{2} \partial_{x} u \partial_{x}^{2} u+h \partial_{x} z\left(\partial_{x} u\right)^{2}+h \partial_{x}^{2} z u \partial_{x} u+\left(\partial_{x} \zeta \partial_{x}^{2} z+\frac{h}{2} \partial_{x}^{3} z\right) u^{2},
$$

and $\mathbb{T}[z, h]=h \mathcal{T}[z, h] \frac{1}{h}$, with for any scalar valued function $w$ :

$$
\mathcal{T}[z, h] w=-\frac{h^{2}}{3} \partial_{x}^{2} w-h \partial_{x} h \partial_{x} w+\left(\partial_{x} \zeta \partial_{x} z+\frac{h}{2} \partial_{x}^{2} z\right) w,
$$

with $z$ a parametrization of the bottom variations, $h=\zeta+h_{0}-z$ referring to the water height (see Fig. 1) and $\alpha$ an optimization parameter.

A compact FVM approach is proposed for the 1d case in [11] and a FVM for a particular 2d flat bottom system in [44]. A pseudo-spectral approach is introduced in [56] and FEM discretization in [53] for the 1d case on flat bottom. To the authors knowledge, the only dG method for GN equations has been recently proposed in [45], for the $1 \mathrm{~d}$ equations with uneven bottom, relying on a centered $\mathrm{dG}$ approach and applications with second-order polynomial approximations. The issue of robustness is however not addressed. In $[7,9,68]$, a high-order accurate hybrid FVM-FDM model is introduced, embedded in a splitting approach. A robust treatment of moving shoreline and well-balancing for motionless steady states are ensured. The $2 \mathrm{~d}$ extension on cartesian meshes has 
recently been performed in [43]. Let us also mention [10] and more recently [63] for a 2D cartesian numerical model based on fully non-linear BT equations of [76]. To our knowledge no arbitrary order dG discretization of the GN equations has been proposed yet, and this is one of the goal of this work.

Another challenging issue is that although the GN equations provide a very good description of waves up to the breaking point, they are not entirely satisfactory as a nearshore flows simulation model. They are indeed unable to handle broken waves. To amend this important limitation, several approaches have been proposed. The most common one is to add an artificial viscous term to the momentum equation, whose role is to account for the energy dissipation that occurs during wave breaking (see for instance [10,12,39] for a related approach). This is also the method chosen in [23], embedded in the nodal-dG method. One of the drawbacks of this approach, as mentioned in [13], is that the computation remains very sensitive to the calibrations of the dissipation parameters.

An alternative approach to handle broken waves, which is becoming very popular, is to switch from the GN to the NSW equations in the vicinity of breaking waves. Indeed, NSW equations actually provide a good description of broken waves [6], which are regarded as shock waves, and can therefore be accurately computed using shockcapturing methods. This switching strategy can be performed in a very simple fashion, provided the dispersive BT or GN equations are written in conservative forms. This approach is used for instance in $[7,38,55,60,63,71]$ where the occurrences of shock waves are accounted for through the use of FVM. Naturally, the method requires the introduction of some criteria devoted to detect waves which are about to break, and eventually to follow broken waves to detect if a "switch-back" to dispersive equations is needed. In [69] a criterium relying on the wave height to water depth ratio is introduced and subsequently used in [70] and [63] for instance. Another criterium based on the momentum gradient is successfully used in [60]. In [68], a relevant combination of the local energy dissipation, front slope and Froude number is proposed. Let us also mention the recent $2 \mathrm{~d}$ approach of [38].

In the present work, we describe a strategy to compute discontinuous-Galerkin approximations of the solutions of some one-dimensional GN equations, including an efficient wave-breaking method. Indeed, the use of such fully nonlinear equations appear as a reasonable compromise between the weakly non-linear equations studied in [30,31] and the highly-dispersive (and computationally costly) three-variables equations investigated in $[22,23]$. Additionally, it is easily possible to extend the range of validity of the GN equations to moderately deep water by the introduction of some optimization parameters, as shown in [9, 43].

A closer look to equations (1) highlights that, if this formulation has real advantages (i.e. it does not involve any third order derivatives, and the presence of the second-order operator $I+\alpha \mathbb{T}[z, h]$ makes the model very stable with respect to high frequency perturbations, see [7]), its main drawback is the time-dependency of this operator, through the water height $h$. Indeed, from a computational point of view, the dG discretization of $I+\alpha \mathbb{T}[z, h]$ with a Local Discontinuous Galerkin approach [15] for higher order derivatives, and the associated linear system resolution, implies a global assembly process, for gathering the local discrete operators into the global one. And this should be done at each time step, or even sub step if high order time marching algorithms are used, together with the corresponding algebraic system resolution.

To overcome this, the strategy recently proposed in [43], and also applied in this work, consists in deriving a new class of GN equations, asymptotically equivalent to the formulation (1) but for which the time dependency of the operator $I+\alpha \mathbb{T}[z, h]$ is removed. The resulting model shares the same level of accuracy with the original GN equations, with respect to the approximation of the full water waves equations, but allows to build the global discrete operator in a pre-processing step. This operator is then used throughout all the computation, leading to dramatic computational savings.

As a consequence, the computational effort can be oriented towards a high order of accuracy in space and time, and the enforcement of some essential robustness properties. An arbitrary order of accuracy in space is obtained through the use of the Legendre polynomials hierarchical basis, and low storage strong-stability preserving Runge-Kutta methods (SSP-RK in the following) are used for the time discretization. The whole 
model is shown to exactly preserve the motionless steady states, thanks to the pre-balanced reformulation of the surface gradient term and suitable interface fluxes [20], and a robust treatment is implemented for the moving shoreline, based on the enforcement of an element-wise water height positivity preservation property, borrowing the recent accuracy-preserving method introduced for the dGM in [79,81].

We lastly introduce an efficient way of handling broken waves, relying on the GN-NSW switching method. The strong super-convergence criteria introduced in [40] is successfully used to detect steep wave fronts and locally suppress the dispersive effects, which can be done in a natural fashion, as the dispersive part of the GN equations is regarded as an additional source term in the NSW equations. The corresponding shock waves are subsequently computed using the natural shock-capturing abilities of the dG approach, combined with an efficient local limiting process.

To summarize, the main features of the $1 \mathrm{~d}$ dG-based GN numerical model described in this work are:

- an improved computational efficiency when compared to the original equations (1), due to the timeindependency of the involved regularizing second order dispersive operator,

- an arbitrary order of spatial accuracy,

- a well-balanced property for the motionless steady states,

- a robust treatment of the shoreline motions,

- an efficient and simple way of handling broken waves, based on a GN/NSW equations switching strategy.

This paper is organized as follows: in a first part we detail the 1d derivation of a new family of GN model, following the strategy detailed above. The second part is devoted to the description of a robust arbitrary order of accuracy $\mathrm{dG}$ approach for this family of model, ensuring the preservation of motionless steady states. The emphasize is put on the discretization of the higher order derivatives. Finally, the accuracy and robustness of the resulting numerical model are assessed through extensive $1 \mathrm{~d}$ numerical validations. Two-dimensional validations are left for future works.

\section{Derivation of THE MODEL}

\subsection{A new class of GN equations}

In this section, we detail the simplified 1d derivation of a new family of GN equations for which the time dependency of the second-order differential operator $I+\alpha \mathbb{T}[z, h]$ is amended. Choosing $h_{0}$ a reference water depth, we recall that $\zeta(x, t)$ stands for the free surface elevation, $z(x)$ the variation of the bottom with respect to the rest state, $u$ the horizontal velocity, and $h(x, t)=\zeta(x, t)+h_{0}-z(x)$ the water height (see Fig. 1).

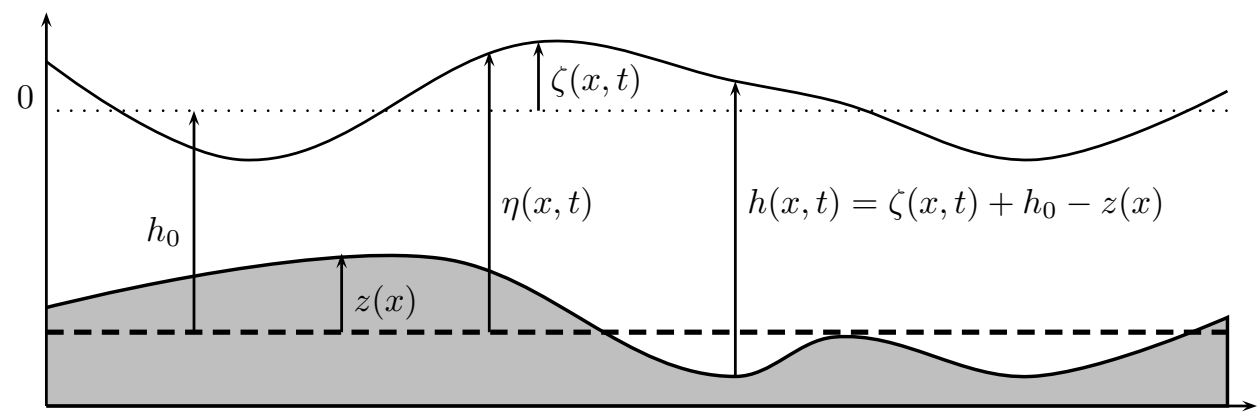

FigURE 1. 1D configuration

Defining $\varepsilon=a / h_{0}$ the nonlinearity parameter, $\mu=h_{0}^{2} / \lambda^{2}$ the shallowness parameter, $\beta=a_{b} / h_{0}$ the bottom variations parameter, with $a_{b}$ the typical bottom deformation amplitude, and choosing $\alpha=1$ for the sake of 
simplicity, the 1d GN equations (1) can be written in their non-dimensionalized formulation :

$$
\left\{\begin{array}{l}
\partial_{t} \zeta+\varepsilon \partial_{x}(h u)=0, \\
{[1+\mu \tilde{\mathbb{T}}[z, h]]\left(\partial_{t}(h u)+\varepsilon \partial_{x}\left(h u^{2}\right)\right)+h \partial_{x} \zeta+\mu \varepsilon h \tilde{\mathcal{Q}}_{1}(u)=0,}
\end{array}\right.
$$

where the non-dimensionalized water depth is $h(x, t)=1+\varepsilon \zeta(x, t)-\beta z(x)$ and the non-dimensionalized differential operators are written as follows:

$$
\begin{aligned}
& \tilde{\mathcal{Q}}_{1}(u)=2 h \partial_{x} h\left(\partial_{x} u\right)^{2}+\frac{4}{3} h^{2} \partial_{x} u \partial_{x}^{2} u+ \\
& \beta\left(h \partial_{x} z\left(\partial_{x} u\right)^{2}+h \partial_{x}^{2} z u \partial_{x} u+\left(\varepsilon \partial_{x} \zeta \partial_{x}^{2} z+\frac{h}{2} \partial_{x}^{3} z\right) u^{2}\right), \\
& \tilde{\mathbb{T}}[z, h]=h \tilde{\mathcal{T}}[z, h] \frac{1}{h},
\end{aligned}
$$

with for any scalar valued function $w$ :

$$
\tilde{\mathcal{T}}[z, h] w=-\frac{h^{2}}{3} \partial_{x}^{2} w-h \partial_{x} h \partial_{x} w+\beta\left(\varepsilon \partial_{x} \zeta \partial_{x} z+\frac{h}{2} \partial_{x}^{2} z\right) w .
$$

Using the fact that

$$
\partial_{t}(h u)+\varepsilon \partial_{x}\left(h u^{2}\right)=-h \partial_{x} \zeta+O(\mu),
$$

we can easily remove the $z$ dependency of $\tilde{\mathbb{T}}[z, h]$. The second equation of (3) is written as follows:

$$
[1+\mu \mathfrak{T}[h]]\left(\partial_{t}(h u)+\varepsilon \partial_{x}\left(h u^{2}\right)\right)+h \partial_{x} \zeta+\varepsilon \mu h \tilde{\mathcal{Q}}_{1}(u)+\mu h \tilde{\mathcal{Q}}_{2}(\zeta)=O\left(\mu^{2}\right),
$$

with

$$
\begin{aligned}
& \mathfrak{T}[h] w=-\frac{h^{3}}{3} \partial_{x}^{2}\left(\frac{w}{h}\right)-h^{2} \partial_{x} h \partial_{x}\left(\frac{w}{h}\right), \\
& \tilde{\mathcal{Q}}_{2}(\zeta)=-\beta\left(\varepsilon \partial_{x} \zeta \partial_{x} z+\frac{h}{2} \partial_{x}^{2} z\right) \partial_{x} \zeta .
\end{aligned}
$$

The last step is to remove the $h$ dependency of the operator $\mathfrak{T}[h]$. This could be achieved following the same substitution (5), and we would obtain a model without any operator to invert, but at the price of loosing the stabilizing effects associated to this inverse operator. To avoid this, let us introduce:

$$
h_{b}=\max \left(h-\varepsilon \zeta, \epsilon_{b}\right),
$$

where $\epsilon_{b}$ is a positive threshold value to be specified later. Observing that we have, for an arbitrary scalar valued function $w$ :

with

$$
\mathfrak{T}[h] w=\mathfrak{T}\left[h_{b}\right] w-\tilde{\mathcal{Q}}_{3}\left[h^{2}-h_{b}^{2}\right] w,
$$

$$
\tilde{\mathcal{Q}}_{3}\left[h^{2}-h_{b}^{2}\right] w=\frac{1}{6} \partial_{x}\left(h^{2}-h_{b}^{2}\right) \partial_{x} w+\frac{h^{2}-h_{b}^{2}}{3} \partial_{x}^{2} w-\frac{1}{6} \partial_{x}^{2}\left(h^{2}-h_{b}^{2}\right) w,
$$

equation (6) can be reformulated as:

$$
\begin{gathered}
{\left[1+\mu \mathfrak{T}\left[h_{b}\right]\right]\left(\partial_{t}(h u)+\varepsilon \partial_{x}\left(h u^{2}\right)\right)-\mu \tilde{\mathcal{Q}}_{3}\left[h^{2}-h_{b}^{2}\right]\left(\partial_{t}(h u)+\varepsilon \partial_{x}\left(h u^{2}\right)\right)} \\
+h \partial_{x} \zeta+\varepsilon \mu h \tilde{\mathcal{Q}}_{1}(u)+\mu h \tilde{\mathcal{Q}}_{2}(\zeta)=O\left(\mu^{2}\right) .
\end{gathered}
$$


Denoting that

$$
\partial_{t}(h u)+\varepsilon \partial_{x}\left(h u^{2}\right)=-\left[1+\mu \mathfrak{T}\left[h_{b}\right]\right]^{-1}\left(h \partial_{x} \zeta\right)+O(\mu),
$$

we obtain the following non-dimensionalized model:

$$
\left\{\begin{array}{l}
\partial_{t} \zeta+\varepsilon \partial_{x}(h u)=0 \\
{\left[1+\mu \mathfrak{T}\left[h_{b}\right]\right]\left(\partial_{t} h u+\partial_{x}\left(h u^{2}\right)\right)+h \partial_{x} \zeta+\varepsilon \mu h \tilde{\mathcal{Q}}_{1}(u)} \\
\quad+\mu h \tilde{\mathcal{Q}}_{2}(\zeta)+\mu \tilde{\mathcal{Q}}_{3}\left[h^{2}-h_{b}^{2}\right]\left(\left[1+\mu \tilde{\mathfrak{T}}\left[h_{b}\right]\right]^{-1}\left(h \partial_{x} \zeta\right)\right)=O\left(\mu^{2}\right)
\end{array}\right.
$$

Switching back to dimensionalized quantities, introducing the optimization parameter $\alpha$ again, and dropping the $O\left(\mu^{2}\right)$, we obtain the following family of $1 \mathrm{~d}$ Green-Naghdi equations with a time-independent operator:

$$
\left\{\begin{array}{l}
\partial_{t} \zeta+\partial_{x}(h u)=0 \\
{\left[1+\alpha \mathfrak{T}\left[h_{b}\right]\right]\left(\partial_{t} h u+\partial_{x}\left(h u^{2}\right)+\frac{\alpha-1}{\alpha} g h \partial_{x} \zeta\right)+\frac{1}{\alpha} g h \partial_{x} \zeta} \\
\quad+h\left(\mathcal{Q}_{1}(u)+g \mathcal{Q}_{2}(\zeta)\right)+g \mathcal{Q}_{3}\left(\left[1+\alpha \mathfrak{T}\left[h_{b}\right]\right]^{-1}\left(g h \partial_{x} \zeta\right)\right)=0
\end{array}\right.
$$

where $h_{b}, \mathcal{Q}_{1}, \mathcal{Q}_{2}$ and $\mathcal{Q}_{3}$ are obtained from (8), (1.1), (7b) and (9) replacing $\varepsilon, \beta$ and $\mu$ by 1 and dropping the $\left[h^{2}-h_{b}^{2}\right]$ in $\tilde{\mathcal{Q}}_{3}\left[h^{2}-h_{b}^{2}\right]$.

Remark 1.1. We refer the reader to [43] for a generalized $2 \mathrm{~d}$ derivation and further optimizations, with a detailed study of the dispersion relation of the corresponding linearized model. Also, a discussion on the stability issues, and in particular on the reason why we substitute relation (11) in (10) instead of (5), is provided.

Remark 1.2. The use of $\epsilon_{b}$ in (8) is only to ensure that the new quantity $h_{b}$ remains positive in dry areas and preserve the regularization properties of $1+\alpha \mathfrak{T}\left[h_{b}\right]$. It obviously does not modify the consistency of the model, and our numerical investigations did not show any dependency of the results with respect to the (reasonable) value of $\epsilon_{b}$. In the validations of $\S 3$ we use $\epsilon_{b}=0.1$.

\subsection{Pre-balanced formulation}

Before describing in details the $\mathrm{dG}$ discretization of (12), let us reformulate the model under the pre-balanced formulation, in order to adapt the FVM well-balanced discretization introduced in [20,48]. The main ideas are, firstly, to use the free surface elevation instead of $h$ as a flow variable, and secondly to introduce an alternative splitting and redistributing of the free surface gradient term $g h \partial_{x} \zeta$, exploiting the deviations from the system's unforced equilibrium. This method was first introduced in [61] for the NSW equations in the framework of FV methods and then extended to handle the occurrence of dry areas in [47,48], where the following splitting is introduced, relying on the use of the total free surface elevation $\eta=h+z$ (see Fig. 1):

$$
g h \partial_{x} \eta=\frac{1}{2} g \partial_{x}\left(\eta^{2}-2 \eta z\right)+g \eta \partial_{x} z
$$

leading to the following formulation of the NSW system:

$$
\partial_{t} \mathbf{w}+\partial_{x} \mathcal{F}(\mathbf{w}, z)=\mathcal{B}(\mathbf{w}, z)
$$

with $\mathbf{w}={ }^{t}(\eta, q), q=h u$ and

$$
\mathcal{F}(\mathbf{w}, z)=\left(\begin{array}{c}
\mathcal{F}_{\eta}(\mathbf{w}) \\
\mathcal{F}_{q}(\mathbf{w}, z)
\end{array}\right)=\left(\begin{array}{c}
q \\
\frac{q^{2}}{\eta-z}+p(\eta, z)
\end{array}\right), \mathcal{B}(\mathbf{w}, z)=\left(\begin{array}{c}
\mathcal{B}_{\eta}(\mathbf{w}) \\
\mathcal{B}_{q}(\mathbf{w}, z)
\end{array}\right)=\left(\begin{array}{c}
0 \\
-g \eta \partial_{x} z
\end{array}\right)
$$


and the hydrostatic pressure term now expressed as:

$$
p(\eta, z)=\frac{1}{2} g \eta(\eta-2 z) .
$$

Simple and robust well-balanced schemes for the NSW equations relying on this pre-balanced formulation are proposed for instance in $[20,74]$. Using dG approximations for NSW equations, we show in the next section that this formulation also provides a natural balance between fluxes and topography source term, provided that the corresponding integral terms are computed exactly. To benefit from this property for the GN equations, we notice that using (13), the second equation of (12) can be equivalently written as follows:

$$
\partial_{t} q+\partial_{x}\left(\frac{q^{2}}{\eta-z}+p\right)+\mathfrak{D}_{c}(\eta, u)=-g \eta \partial_{x} z
$$

with

$$
\begin{aligned}
& \mathfrak{D}_{c}(\eta, u)=\left[1+\alpha \mathfrak{T}\left[h_{b}\right]\right]^{-1}\left(\frac{1}{\alpha} g h \partial_{x} \eta+h\left(\mathcal{Q}_{1}(u)+g \mathcal{Q}_{2}(\eta)\right)\right. \\
& \left.\quad+g \mathcal{Q}_{3}\left(\left[1+\alpha \mathfrak{T}\left[h_{b}\right]\right]^{-1}\left(g h \partial_{x} \eta\right)\right)\right)-\frac{1}{\alpha} g h \partial_{x} \eta
\end{aligned}
$$

where $u=\frac{q}{\eta-z}$ is kept in the writing of $\mathfrak{D}_{c}(\eta, u)$ for the sake of simplicity.

Remark 1.3. The original system (1) can of course also be written as follows:

$$
\partial_{t} q+\partial_{x}\left(\frac{q^{2}}{\eta-z}+p\right)+\mathfrak{D}_{o}(\eta, u)=-g \eta \partial_{x} z,
$$

with

$$
\mathfrak{D}_{o}(\eta, u)=[1+\alpha \mathbb{T}[z, h]]^{-1}\left(\frac{1}{\alpha} g h \partial_{x} \eta+h \mathcal{Q}_{1}(u)\right)-\frac{1}{\alpha} g h \partial_{x} \eta .
$$

From this, equations (1) and (12) are nothing but the pre-balanced NSW equations (14) supplemented by a dispersive source term:

$$
\partial_{t} \mathbf{w}+\partial_{x} \mathcal{F}(\mathbf{w}, z)=\mathcal{B}(\mathbf{w}, z)+\mathfrak{D}(\mathbf{w})
$$

with

$$
\begin{array}{ll}
\operatorname{model}(1) \quad: \quad \mathfrak{D}(\mathbf{w})=\left(\begin{array}{c}
0 \\
-\mathfrak{D}_{o}(\eta, u)
\end{array}\right) \\
\operatorname{model}(12) \quad: \quad \mathfrak{D}(\mathbf{w})=\left(\begin{array}{c}
0 \\
-\mathfrak{D}_{c}(\eta, u)
\end{array}\right) .
\end{array}
$$

\section{Discontinuous Galerkin Discretization}

To implement a discontinuous Galerkin scheme, let $\mathcal{P}_{\mathfrak{h}}$ be a partition of the computational domain $\Omega=[L, R]$ into $N_{e}$ non-overlapping elements, denoted $\mathcal{E}^{j}, 1 \leq j \leq N_{e}$. The element $\mathcal{E}^{j}=\left[x_{l}^{j}, x_{r}^{j}\right]$ has a length $\left|\mathcal{E}^{j}\right|$, a boundary $\partial \mathcal{E}^{j}$ (reduced to the 2 boundary nodes $x_{l}^{j}$ and $x_{r}^{j}$ ) and a unit outward normal $\hat{\mathbf{n}}^{\mathbf{j}}$ (reduced to +1 and -1 respectively at the right and the left boundary). Let $x$ be the coordinate in $\Omega$ and we denote $\mathfrak{h}=\max _{1 \leq j \leq N_{e}}\left|\mathcal{E}^{j}\right|$. We aim at computing an approximated solution, denoted $\mathbf{w}_{\mathfrak{h}}=\left(\eta_{\mathfrak{h}}, q_{\mathfrak{h}}\right)$, on this partition. Let us define

$$
\mathcal{V}_{\mathfrak{h}}:=\left\{v \in L^{2}(\Omega) \mid \forall \mathcal{E} \in \mathcal{P}_{\mathfrak{h}}, v_{\mid \mathcal{E}} \in \mathbb{P}^{N}(\mathcal{E})\right\},
$$


where $\mathbb{P}^{N}(\mathcal{E})$ denotes the space of polynomials in $\mathcal{E}$ of degree at most $N$. We consider only the new model (12) but the subsequent weak formulation and discretization can of course be straightforwardly applied to the original model (1).

\subsection{Weak formulation}

Due to the number of terms and derivatives (up to $3^{\text {rd }}$ order) involved in the dispersive source term $\mathfrak{D}_{c}^{p b}\left[h_{b}\right](\eta, u)$, trying to write equation (16) as a first-order system, with the use of several auxiliary variables, would only bring up some unnecessary complexity in the formulation. Instead, we reformulate (16) as follows:

$$
\begin{aligned}
& \partial_{t} \eta+\partial_{x} q=0 \\
& \partial_{t} q+\partial_{x}\left(\frac{q^{2}}{\eta-z}+p\right)+\mathcal{H}=-g \eta \partial_{x} z
\end{aligned}
$$

with $\mathcal{H}$ is an auxiliary scalar valued variable, obtained as the solution of the following auxiliary problem:

$$
\begin{aligned}
& {\left[1+\alpha \mathfrak{T}\left[h_{b}\right]\right]\left(\mathcal{H}+\frac{1}{\alpha} g h \partial_{x} \eta\right)=\mathcal{K},} \\
& \mathcal{K}=\frac{1}{\alpha} g h \partial_{x} \eta+h\left(\mathcal{Q}_{1}(u)+g \mathcal{Q}_{2}(\eta)\right)+g \mathcal{Q}_{3}(\mathcal{L}), \\
& {\left[1+\alpha \mathfrak{T}\left[h_{b}\right]\right] \mathcal{L}=g h \partial_{x} \eta .}
\end{aligned}
$$

From this, a weak formulation of the problem (23) is obtained by multiplying by test functions $\left(\phi_{\mathfrak{h}}, \pi_{\mathfrak{h}}\right) \in\left(\mathcal{V}_{\mathfrak{h}}\right)^{2}$ and integrating over a given element $\mathcal{E}^{j}$; the flux term is integrated by parts. We obtain: find $\mathbf{w}_{\mathfrak{h}}=\left(\eta_{\mathfrak{h}}, q_{\mathfrak{h}}\right) \in$ $\left(\mathcal{V}_{\mathfrak{h}}\right)^{2}$ such that, $\forall\left(\phi_{\mathfrak{h}}, \pi_{\mathfrak{h}}\right) \in\left(\mathcal{V}_{\mathfrak{h}}\right)^{2}$, and $\forall \mathcal{E}^{j} \in \mathcal{P}_{\mathfrak{h}}$ we have:

$$
\begin{gathered}
\int_{\mathcal{E}^{j}} \partial_{t} \eta_{\mathfrak{h}} \phi_{\mathfrak{h}} d x-\int_{\mathcal{E}^{j}} q_{\mathfrak{h}} \phi_{\mathfrak{h}}^{\prime} d x+\left[q_{\mathfrak{h}} \phi_{\mathfrak{h}}\right]_{x_{l}^{j}}^{x_{r}^{j}}=0 \\
\int_{\mathcal{E}^{j}} \partial_{t} q_{\mathfrak{h}} \pi_{\mathfrak{h}} d x-\int_{\mathcal{E}^{j}} \mathcal{F}_{q}\left(\mathbf{w}_{\mathfrak{h}}, z_{\mathfrak{h}}\right) \pi_{\mathfrak{h}}^{\prime} d x+\left[\mathcal{F}_{q}\left(\mathbf{w}_{\mathfrak{h}}, z_{\mathfrak{h}}\right) \pi_{\mathfrak{h}}\right]_{x_{l}^{j}}^{x_{r}^{j}}=\int_{\mathcal{E}^{j}} \mathcal{B}_{q}\left(\mathbf{w}_{\mathfrak{h}}, z_{\mathfrak{h}}\right) \pi_{\mathfrak{h}} d x-\int_{\mathcal{E}^{j}} \mathcal{H}_{\mathfrak{h}} \pi_{\mathfrak{h}} d x
\end{gathered}
$$

\subsection{Semi-discrete formulation}

On each element $\mathcal{E}^{j}$, we write $\forall x \in \mathcal{E}^{j}, \forall t \in\left[0, t_{\text {max }}\right]$ :

$$
\eta_{\mathfrak{h} \mid \mathcal{E}^{j}}(x, t)=\sum_{i=1}^{N_{d}} \tilde{\eta}_{i}^{j}(t) \theta_{i}^{j}(x), \quad \text { and } \quad q_{\mathfrak{h} \mid \mathcal{E}^{j}}(x, t)=\sum_{i=1}^{N_{d}} \tilde{q}_{i}^{j}(t) \theta_{i}^{j}(x)
$$

where $N_{d}=N+1$ is the number of freedom degrees per element, $\left\{\theta_{i}^{j}\right\}_{i=1 \ldots N_{d}}$ is a polynomial expansion basis for $\mathbb{P}^{N}\left(\mathcal{E}^{j}\right)$, and

$$
\tilde{\eta}^{j}={ }^{t}\left(\tilde{\eta}_{1}^{j}(t), \ldots, \tilde{\eta}_{N_{d}}^{j}(t)\right), \quad \tilde{q}^{j}={ }^{t}\left(\tilde{q}_{1}^{j}(t), \ldots, \tilde{q}_{N_{d}}^{j}(t)\right)
$$

are the local expansion coefficients vectors. In the following, $\left\{\theta_{i}^{j}\right\}_{i=1, \ldots, N_{d}}$ refers to the local Lagrange interpolating polynomial basis on the element $\mathcal{E}^{j}$, defined on the Legendre-Gauss-Lobatto (LGL) set of $N_{d}$ nodes (a nodal-element basis). We consider the local polynomial expansion for the topography parameterization

$$
z_{\mathfrak{h} \mid \mathcal{E}^{j}}(x)=\sum_{i=1}^{N_{d}} \tilde{z}_{i}^{j} \theta_{i}^{j}(x)
$$


and we assume that a local expansion is available for $\mathcal{H}_{\mathfrak{h}}$, given by:

$$
\tilde{\mathcal{H}}^{j}={ }^{t}\left(\tilde{\mathcal{H}}_{1}^{j}(t), \ldots, \tilde{\mathcal{H}}_{N_{d}}^{j}(t)\right) .
$$

Injecting these expansions into the weak formulation (25)-(26), replacing the test functions $\left(\phi_{\mathfrak{h}}, \pi_{\mathfrak{h}}\right)$ by the local basis functions and classically substituting numerical flux functions $\widehat{q}$ and $\widehat{\mathcal{F}}$ to the discontinuous intercell flux functions arising in the interface terms, we obtain the semi-discrete formulation of our dG approximation:

$$
\begin{gathered}
\sum_{i=1}^{N_{d}} \frac{d}{d t} \tilde{\eta}_{i}^{j}(t) \mathbf{M}_{i k}^{j}-\sum_{i=1}^{N_{d}} \tilde{q}_{i}^{j}(t) \mathbf{S}_{i k}^{j}+\left[\widehat{q} \theta_{k}^{j}\right]_{x_{l}^{j}}^{x_{r}^{j}}=0, \quad 1 \leq k \leq N p, \\
\sum_{i=1}^{N_{d}} \frac{d}{d t} \tilde{q}_{i}^{j}(t) \mathbf{M}_{i k}^{j}-\int_{\mathcal{E}^{j}} \mathcal{F}_{q}\left(\mathbf{w}_{\mathfrak{h}}, z_{\mathfrak{h}}\right) \frac{d}{d x} \theta_{k}^{j} d x+\left[\widehat{\mathcal{F}} \theta_{k}^{j}\right]_{x_{l}^{j}}^{x_{r}^{j}}=\int_{\mathcal{E}^{j}} \mathcal{B}_{q}\left(\mathbf{w}_{\mathfrak{h}}, z_{\mathfrak{h}}\right) \theta_{k}^{j} d x-\sum_{i=1}^{N_{d}} \tilde{\mathcal{H}}_{i}^{j}(t) \mathbf{M}_{i k}^{j}, \quad 1 \leq k \leq N p,
\end{gathered}
$$

where

$$
\mathbf{M}_{i k}^{j}=\int_{x_{l}^{j}}^{x_{r}^{j}} \theta_{i}^{j}(x) \theta_{k}^{j}(x) d x, \text { and } \mathbf{S}_{i k}^{j}=\int_{x_{l}^{j}}^{x_{r}^{j}} \theta_{i}^{j}(x) \frac{d}{d x} \theta_{k}^{j}(x) d x,
$$

are respectively the local mass and stiffness matrix coefficients on $\mathcal{E}^{j}$.

Remark 2.1. As $\left\{\theta_{i}^{j}\right\}_{i=1 \ldots N_{d}}$ refers to the Lagrange interpolating polynomials, the expansion coefficients represent the values of the considered approximated quantity at the Gauss-Legendre-Lobatto set of nodes. The local expansion for the topography parameterization $\tilde{z}^{j}={ }^{t}\left(\tilde{z}_{1}^{j}, \ldots, \tilde{z}_{N_{d}}^{j}\right)$ is therefore obtained just by "reading" the values of $z$ at the LGL nodes.

Remark 2.2. In (29), the integrals $\int_{\mathcal{E}^{j}} \mathcal{F}_{q}\left(\mathbf{w}_{\mathfrak{h}}, z_{\mathfrak{h}}\right) \frac{d}{d x} \theta_{k}^{j} d x$ and $\int_{\mathcal{E}^{j}} \mathcal{B}_{q}\left(\mathbf{w}_{\mathfrak{h}}, z_{\mathfrak{h}}\right) \theta_{k}^{j} d x$ have to be computed exactly when motionless steady states are reached, with a suitable quadrature formula, in order to obtain a well-balanced scheme, as shown in $\S 2.5$. On the other hand, the computation of $\tilde{\mathcal{H}}^{j}$, which involves higher order derivatives, is done directly, in a collocation way. This is temporarily postponed to $§ 2.3$.

Remark 2.3. At a given interface, the computation of the exchanging flux term $\widehat{q}$ and $\widehat{\mathcal{F}}$ is classically performed with numerical flux functions $F^{\eta}$ and $F^{q}$, which are Lipschitz continuous, monotone increasing with respect to their first argument, monotone decreasing with respect to their second argument and consistent with the exact flux $\mathcal{F}(\mathbf{w}, z)={ }^{t}\left(\mathcal{F}_{\eta}(\mathbf{w}), \mathcal{F}_{q}(\mathbf{w}, z)\right)$. To ensure the positivity of the water height, we also need to use a water height positivity preserving flux (see §2.7). We use the global Lax-Friedrichs flux in the numerical validations of $\S 3$. These interface fluxes computations, carefully chosen to ensure the preservation of motionless steady-states, are detailed in $\S 2.5$.

\subsection{High-order derivatives and dispersive terms computation}

We are now left with the resolution of systems (24a)-(24c), to compute the expansion $\tilde{\mathcal{H}}$. Although the semidiscrete formulation (28)-(29) could appear purely local, the computation of the dispersive term expansions $\tilde{\mathcal{H}}$ requires a global assembly process, for gathering the local discrete operators into a global one, in the computation of the inverse of the operator $1+\alpha \mathfrak{T}\left[h_{b}\right]$. Additionally, this computation involves the discrete approximation of space derivatives up to $3^{\text {rd }}$ order. We use the Local Discontinuous Galerkin (LDG) approach [15] to compute these derivatives.

To illustrate this procedure, let consider the following $2^{\text {nd }}$ order equation, for an arbitrary scalar valued function $w$ :

$$
\ell-\partial_{x}^{2} w=0
$$

reformulated as a set of two coupled $1^{\text {st }}$ order equation using an auxiliary variable $v$ :

$$
v+\partial_{x} w=0 \quad, \quad \ell+\partial_{x} v=0 .
$$


Then, multiplying by a test function $\phi_{\mathfrak{h}} \in \mathcal{V}_{\mathfrak{h}}$ and integrating over an element $\mathcal{C}^{j}$, we have the associated weak formulation:

$$
\begin{array}{r}
\int_{x_{l}^{j}}^{x_{r}^{j}} v \phi_{\mathfrak{h}}-\int_{x_{l}^{j}}^{x_{r}^{j}} w \phi_{\mathfrak{h}}^{\prime}+\widehat{w}_{r} \phi_{\mathfrak{h}}\left(x_{r}^{j}\right)-\widehat{w}_{l} \phi_{\mathfrak{h}}\left(x_{l}^{j}\right)=0, \\
\int_{x_{l}^{j}}^{x_{r}^{j}} \ell \phi_{\mathfrak{h}}-\int_{x_{l}^{j}}^{x_{r}^{j}} v \phi_{\mathfrak{h}}^{\prime}+\widehat{v}_{r} \phi_{\mathfrak{h}}\left(x_{r}^{j}\right)-\widehat{v}_{l} \phi_{\mathfrak{h}}\left(x_{l}^{j}\right)=0 .
\end{array}
$$

To define the exchanging fluxes $\widehat{w}$ and $\widehat{v}$ at a given interface, we use the following fluxes [14]:

$$
\begin{aligned}
& \widehat{w}=\{w\}-\xi \llbracket w \rrbracket, \\
& \widehat{v}=\{v\}+\nu \llbracket v \rrbracket+\frac{\lambda}{\mathfrak{h}} \llbracket w \rrbracket,
\end{aligned}
$$

with the interface average $\{w\}=\left(w^{+}+w^{-}\right) / 2$ and jump $\llbracket w \rrbracket=\left(w^{+}-w^{-}\right) / 2, w^{-}$and $w^{ \pm}$respectively the right and left interface values of $w$ (and similar notations for $v$ ). Taking $\xi=\nu=\lambda=0$ gives the centered Bassi and Rebay (BR) fluxes [4] which usually provide sub-optimal convergence rates for odd values of $N$, while the choice $\xi=\nu=1$, corresponding to the Cockburn and Shu alternate upwind discretization (refers to as LDG flux in the following), may allow to recover the optimal convergence order.

Remark 2.4. Note that $\frac{\lambda}{\mathfrak{h}} \llbracket w \rrbracket$ is a penalization term, with a $O\left(\mathfrak{h}^{-1}\right)$ scaling. Taking for instance $\xi=\nu=0$ and $\lambda \neq 0$ gives the stabilized centered fluxes (sBR), sometimes helping to recover optimal convergence rates. In the numerical validation of $\S 3$, we only investigate and compare the BR and LDG choices. Note that extensive comparisons of fluxes including their stabilized versions and the impact of the coefficient $\lambda$ are performed in [31] for weakly non-linear BT equations.

Let us now come back to (32). At the discrete level, starting from the $N_{e}$ expansion coefficients vectors $\left\{\tilde{w}^{j}\right\}_{i=1 . . N_{e}}$, which are gathered in a $N_{d} \times N_{e}$ vector $\tilde{W}={ }^{t}\left(\tilde{w}^{1}, \ldots, \tilde{w}^{N_{e}}\right)$, we aim at computing the $N_{d} \times N_{e}$ vector $\tilde{L}={ }^{t}\left(\tilde{\ell}^{1}, \ldots, \tilde{\ell}^{N_{e}}\right)$ of expansion coefficients for the $2^{\text {nd }}$ order derivative $\partial_{x}^{2} w$.

This is done globally, using (33) to build the differentiation matrices, that also account for exchanging interface fluxes. Injecting $\tilde{w}^{j}$ into (32) and replacing $\phi_{\mathfrak{h}}$ by the local basis functions $\left\{\theta_{i}^{j}\right\}_{i=1}^{N_{d}}$, for all elements $\left\{\mathcal{E}^{j}\right\}_{i=1 . . N_{e}}$, we obtain the global discrete formulation:

$$
\begin{aligned}
& \mathbb{M} \tilde{V}=\mathbb{S} \tilde{W}-(\mathbb{E}-\xi \mathbb{F}) \tilde{W} \\
& \mathbb{M} \tilde{L}=\mathbb{S} \tilde{V}-(\mathbb{E}+\nu \mathbb{F}) \tilde{V}-\frac{\lambda}{\mathfrak{h}} \mathbb{F} \tilde{W}
\end{aligned}
$$

where the square $N_{d} \times N_{e}$ global mass and stiffness matrices $\mathbb{M}$ and $\mathbb{S}$ have a block-diagonal structure

$$
\mathbb{M}=\left(\begin{array}{lll}
\mathbf{M}^{1} & & \\
& \ddots & \\
& & \mathbf{M}^{N_{e}}
\end{array}\right) \quad, \quad \mathbb{S}=\left(\begin{array}{lll}
\mathbf{S}^{1} & & \\
& \ddots & \\
& & \mathbf{S}^{N_{e}}
\end{array}\right)
$$

and the matrices $\mathbb{E}$ and $\mathbb{F}$ accounting for the inter-element exchanging fluxes have the following structure: 


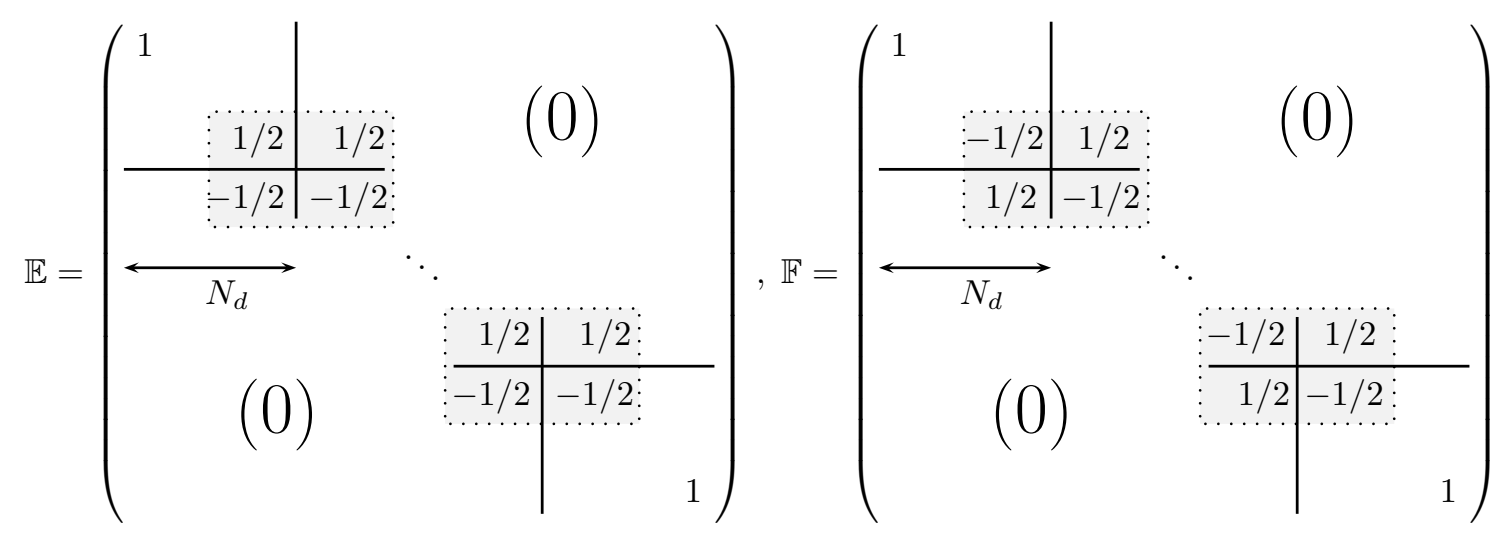

Note that the issue of boundary conditions is postponed to $\S 2.8$. We obtain a global discrete formulation of the first and second order derivative operators, based on the LDG approach (33) :

$$
\tilde{V}=\mathbb{D}_{x} \tilde{W} \quad, \quad \tilde{L}=\mathbb{D}_{x}^{2} \tilde{W}
$$

with

$$
\begin{aligned}
& \mathbb{D}_{x}=\mathbb{M}^{-1}(\mathbb{S}-\mathbb{E}+\xi \mathbb{F}) \\
& \mathbb{D}_{x}^{2}=\mathbb{M}^{-1}\left((\mathbb{S}-\mathbb{E}-\nu \mathbb{F}) \mathbb{D}_{x}-\frac{\lambda}{\mathfrak{h}} \mathbb{F}\right)
\end{aligned}
$$

A similar construction can be performed for the $3^{\text {rd }}$ order derivatives. Using these global differentiation matrices, we are now able to approximate all the derivatives occurring in (24a)-(24c). The nonlinear products are treated directly, in a collocation manner, inspired from [22].

We can also build the global square $N_{d} \times N_{e}$ matrix of the discrete version of $1+\alpha \mathfrak{T}\left[h_{b}\right]$. For instance, considering the simplified flat bottom case $\left(h_{b}=h_{0}\right)$, we approximate the corresponding operator as follows:

$$
1+\alpha \mathfrak{T}\left[h_{0}\right]=1-\alpha \frac{h_{0}^{2}}{3} \partial_{x}^{2} \Rightarrow I-\alpha \frac{h_{0}^{2}}{3} \mathbb{D}_{x}^{2},
$$

where $I$ is the $N_{d} \times N_{e}$ identity matrix. The locality of the LDG approach results in a sparse block-structure matrix (the BR flux leads to a 10 elements stencil for a $3^{r d}$ order derivative, while a 6 elements stencil is obtained with the LDG flux), which is stored in a sparse format and LU-factorized at the beginning of the computation, in a pre-processing step. For the validations of $\S(3)$, the factorization and the resolution of the resulting triangular linear systems are performed using the unsymmetric multi-frontal method [17].

Remark 2.5. The same approach can of course also be used for the original model (1), the only difference being that the $1+\alpha \mathfrak{T}[z, h]$ matrix has to be build and factorized at each time step (or substep).

Remark 2.6. The use of direct interpolation/collocation methods for the computation of the nonlinear products in the dispersive source terms reduces the computational cost but can generate some aliasing which deteriorates the solution quality and can lead to instabilities. To amend this, we use the stabilization filtering method (mild nodal filter), as suggested in [22]. 


\subsection{Time discretization}

We use the method of lines, based on Strong Stability Preserving Runge-Kutta (RK-SSP) schemes [33], as time marching algorithms. Up to $N=3$, RK-SSP schemes of order $N+1$ are considered, to ensure equal orders of convergence in space and time. A $4^{t h}$ order SSP-RK scheme is used for $N \geq 3$. At the fully discrete level, we denote $\Delta_{t}^{n}$ the time step used to advance from discrete time value $t^{n}$ to $t^{n+1}$, and the local polynomial expansions at the discrete time $t^{n}$ as follows:

$$
\eta_{\mathfrak{h} \mid \mathcal{E}^{j}}^{n}(x)=\sum_{i=1}^{N_{d}} \tilde{\eta}_{i}^{j, n} \theta_{i}^{j}(x), \quad \text { and } \quad q_{\mathfrak{h} \mid \mathcal{E}^{j}}^{n}(x)=\sum_{i=1}^{N_{d}} \tilde{q}_{i}^{j, n} \theta_{i}^{j}(x),
$$

with the corresponding expansion vectors :

$$
\tilde{\eta}^{j, n}={ }^{t}\left(\tilde{\eta}_{1}^{j, n}, \ldots, \tilde{\eta}_{N_{d}}^{j, n}\right), \quad \tilde{q}^{j, n}={ }^{t}\left(\tilde{q}_{1}^{j, n}, \ldots, \tilde{q}_{N_{d}}^{j, n}\right) .
$$

\subsection{Preservation of motionless steady states}

In this section, we detail the computation of the interface fluxes $\left[\widehat{\mathcal{F}} \theta_{k}^{j}\right]_{x_{l}^{j}}^{x_{j}^{j}}$ appearing in the semi-discrete local discretization (29). To ensure the preservation of motionless steady states, we adapt to the dG framework the method introduced in $[20,47]$ for the FVM discretization of the pre-balanced SW equations. The following scheme can also be regarded as the adaptation to the pre-balanced formulation of the ideas introduced in [77]. Let us introduce the simplified notations:

$$
\begin{array}{llll}
\mathbf{w}_{r}^{-}=\mathbf{w}_{\mathfrak{h}}\left(x_{r}^{j}\right), & z_{r}^{-}=z_{\mathfrak{h}}\left(x_{r}^{j}\right), & \mathbf{w}_{r}^{+}=\mathbf{w}_{\mathfrak{h}}\left(x_{l}^{j+1}\right), & z_{r}^{+}=z_{\mathfrak{h}}\left(x_{l}^{j+1}\right), \\
\mathbf{w}_{l}^{-}=\mathbf{w}_{\mathfrak{h}}\left(x_{l}^{j}\right), & z_{l}^{-}=z_{\mathfrak{h}}\left(x_{l}^{j}\right), & \mathbf{w}_{l}^{+}=\mathbf{w}_{\mathfrak{h}}\left(x_{r}^{j-1}\right), & z_{l}^{+}=z_{\mathfrak{h}}\left(x_{r}^{j-1}\right),
\end{array}
$$

(at the left and right boundaries of $\mathcal{E}^{j}$, the superscripts ${ }^{-}$and ${ }^{+}$stand respectively for the interior and exterior values). Dropping the subscripts, we define at each boundaries :

$$
\begin{array}{ll}
z^{*}=\max \left(z^{-}, z^{+}\right), & \breve{z}=z^{*}-\max \left(0, z^{*}-\eta^{-}\right), \\
\breve{h}^{ \pm}=\max \left(0, \eta^{ \pm}-z^{*}\right), & \breve{\eta}^{ \pm}=\breve{h}^{ \pm}+\breve{z},
\end{array}
$$

leading to new interior and exterior values:

$$
\breve{\mathbf{w}}^{-}=\left(\breve{\eta}^{-}, \frac{\breve{h}^{-}}{h^{-}} q^{-}\right) \text {, and } \breve{\mathbf{w}}^{+}=\left(\breve{\eta}^{+}, \frac{\breve{h}^{+}}{h^{+}} q^{+}\right)
$$

with $h^{ \pm}=\eta^{ \pm}-z^{ \pm}$. Then, the interface fluxes in (28)-(29) are computed as follows:

$$
\begin{aligned}
& \widehat{q}=F_{\eta}\left(\breve{\mathbf{w}}^{-}, \breve{\mathbf{w}}^{+}\right), \\
& \widehat{\mathcal{F}}=F_{q}\left(\breve{\mathbf{w}}^{-}, \breve{\mathbf{w}}^{+}, \breve{z}, \breve{z}\right)-g \breve{\eta}^{-}\left(z^{-}-\breve{z}\right),
\end{aligned}
$$

where $F={ }^{t}\left(F_{\eta}, F_{q}\right)$ is the Lax-Friedrich flux, defined as follows for two arbitrary interface states $\mathbf{w}_{l}, \mathbf{w}_{r}$ :

$$
\begin{aligned}
& F_{\eta}\left(\mathbf{w}_{l}, \mathbf{w}_{r}\right)=\frac{1}{2}\left(\left(q_{l}+q_{r}\right)-\alpha_{0}\left(\eta_{r}-\eta_{l}\right) \hat{\mathbf{n}}\right), \\
& F_{q}\left(\mathbf{w}_{l}, \mathbf{w}_{r}, z_{l}, z_{r}\right)=\frac{1}{2}\left(\left(\mathcal{F}_{q}\left(\mathbf{w}_{l}, z_{l}\right)+\mathcal{F}_{q}\left(\mathbf{w}_{r}, z_{r}\right)\right)-\alpha_{0}\left(q_{r}-q_{l}\right) \hat{\mathbf{n}}\right),
\end{aligned}
$$


with $\alpha_{0}=\max _{1 \leq j \leq N_{e}}\left(\max _{\partial \mathcal{E}^{j}}(|u|+\sqrt{g(\eta-z)})\right)$ and $\hat{\mathbf{n}}$ the outward normal of the element. We point out that the modified fluxes (46)-(47) induce perturbations of order $N+1$ when compared to the traditional interface fluxes $F_{\eta}\left(\mathbf{w}^{-}, \mathbf{w}^{+}\right)$and $F_{q}\left(\mathbf{w}^{-}, \mathbf{w}^{+}, z^{-}, z^{+}\right)$and do not modify the consistency and convergence.

Remark 2.7. $F$ can be of course replaced by any monotone and positive preserving numerical flux function, like the HLL flux [72] or the VFRoe-relaxation flux [5].

Remark 2.8. In (45), we implicitly assumed that we have $h^{ \pm}>0$. The case $h^{ \pm}=0$ (or numerically $h^{ \pm}<\epsilon$ ) is classically handled by setting the corresponding values of $u^{ \pm}$and $q^{ \pm}$to 0 .

Proposition 2.9. The $d G$ scheme (28)-(29), with the interface fluxes defined in (46)-(47), preserves the motionless steady states.

Proof. We adapt the ideas of [77] to the pre-balanced formulation (20). We want to show that

$$
\frac{d}{d t} \tilde{\eta}^{j}(t)=0 \text { and } \frac{d}{d t} \tilde{q}^{j}(t)=0, \text { for } 1 \leq j \leq N_{e}
$$

If a motionless steady state is reached, that is

$$
\mathbf{w}_{\mathfrak{h}}={ }^{t}\left(\eta_{0}, 0\right),
$$

we have $\tilde{q}^{j}(t)=0$ on each element and using (28), $\frac{d}{d t} \tilde{\eta}^{j}(t)=0$ obviously holds. For the second equation (29), we need the residue

$$
R=-\int_{\mathcal{E}^{j}} \mathcal{F}_{q}\left(\mathbf{w}_{\mathfrak{h}}, z_{\mathfrak{h}}\right) \frac{d}{d x} \theta_{k}^{j} d x+\left[\widehat{\mathcal{F}} \theta_{k}^{j}\right]_{x_{l}^{j}}^{x_{j}^{j}}+g \int_{\mathcal{E}^{j}} \eta_{\mathfrak{h}} \frac{d}{d x} z_{\mathfrak{h}} \theta_{k}^{j} d x+\sum_{i=1}^{N_{d}} \tilde{\mathcal{H}}_{i}^{j}(t) \mathbf{M}_{i k}^{j}
$$

to vanish when a motionless steady state is reached, for $1 \leq k \leq N_{d}$ and $1 \leq j \leq N_{e}$. We first notice that the definition of the differential operators $\left\{\mathcal{Q}_{i}\right\}_{1 \leq i \leq 3}$, combined with (50), directly leads to $\mathcal{H}\left(\mathbf{w}_{\mathfrak{h}}\right)=0$ at the discrete level, as a solution of (24a)-(24b)-(24c).

When (50) holds, (43)-(44)-(45) leads to $\breve{\eta}^{-}=\breve{\eta}^{+}=\eta_{0}$ for both interfaces, and using (47), the interface flux $\widehat{\mathcal{F}}\left(x_{r}^{j}\right)$ reduces to

$$
\widehat{\mathcal{F}}\left(x_{r}^{j}\right)=\frac{g}{2}\left(\eta_{0}^{2}-2 \eta_{0} \breve{z}_{r}\right)-g \eta_{0}\left(z_{r}^{-}-\breve{z}_{r}\right)=\frac{g}{2}\left(\eta_{0}^{2}-2 \eta_{0} z_{r}^{-}\right)=\mathcal{F}_{q}\left(\mathbf{w}_{r}^{-}, z_{r}^{-}\right) .
$$

We show in a similar way that, at the left boundary, $\widehat{\mathcal{F}}\left(x_{l}^{j}\right)=\mathcal{F}_{q}\left(\mathbf{w}_{l}^{-}, z_{l}^{-}\right)$. Consequently we have,

$$
R=\int_{\mathcal{E}^{j}} \partial_{x}\left(\mathcal{F}_{q}\left(\mathbf{w}_{\mathfrak{h}}, z_{\mathfrak{h}}\right)\right) \theta_{k}^{j} d x+g \int_{\mathcal{E}^{j}} \eta_{\mathfrak{h}} \frac{d}{d x} z_{\mathfrak{h}} \theta_{k}^{j} d x=0
$$

as $\mathbf{w}_{\mathfrak{h}}$ is a steady state solution, leading to $\partial_{x}\left(\mathcal{F}_{q}\left(\mathbf{w}_{\mathfrak{h}}, z_{\mathfrak{h}}\right)\right)=-g \eta_{\mathfrak{h}} \frac{d}{d x} z_{\mathfrak{h}}$, and the integral terms are computed exactly.

\subsection{Handling broken waves and limiting strategy}

We now broach the issue of broken waves detection and give further details on the switching strategy adopted in this work. As stated in the introductory lines, we can find several methods in the literature, since the last few years, that allow to handle broken waves in BT equations. They all rely on an accurate detection of potential instability regions, near the breaking point, in which the limit of validity of the BT equations is reached. We propose in this work to use a purely numerical criteria to detect such area, using the ideas of the popular discontinuity detector of [40]. This criteria is based on a strong superconvergence property of the dG method 


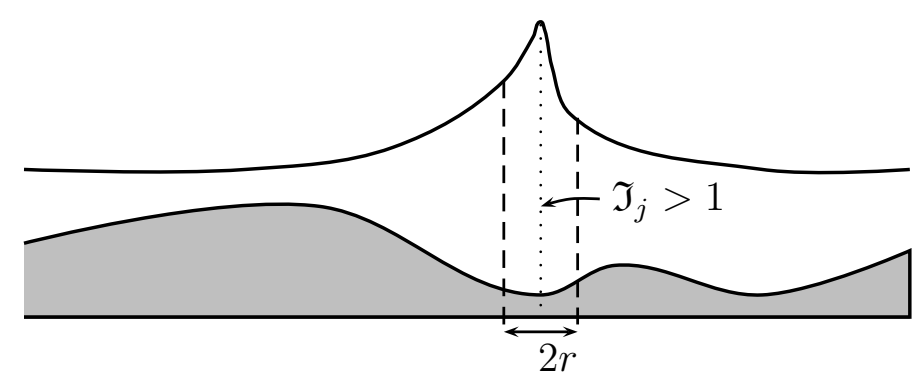

FIGURE 2. Example of breaking area

at the outflow boundary of each element in smooth regions of the flow. More precisely, for a given element $\mathcal{E}^{j}$, we recover the values $\tilde{h}^{j}=\tilde{\eta}^{j}-\tilde{z}^{j}$, and compute the following quantity:

$$
\mathfrak{I}_{j}=\frac{\Delta^{-}\left|\tilde{h}_{1}^{j}-\tilde{h}_{N_{d}}^{j-1}\right|+\Delta^{+}\left|\tilde{h}_{N_{d}}^{j}-\tilde{h}_{1}^{j+1}\right|}{\left|\mathcal{E}_{j}\right|^{\frac{N+1}{2}}\left\|\tilde{h}^{j}\right\|_{\infty}},
$$

where

$$
\Delta^{-}=\left\{\begin{array}{ll}
1 & \text { if } \tilde{q}_{1}^{j} \geq 0, \\
0 & \text { otherwise. }
\end{array} \quad, \quad \Delta^{+}= \begin{cases}1 & \text { if } \tilde{q}_{N_{d}}^{j} \leq 0, \\
0 & \text { otherwise }\end{cases}\right.
$$

Waves about to break are identified in elements such that $\mathfrak{I}_{j}>1$ (called troubled elements, following [58]) and we locally suppress the dispersive term in such elements (i.e. we locally switch to the NSW equations). After breaking, the wave fronts are handled as shocks by the NSW equations and only the hyperbolic part of the equations is solved for the wave fronts. The breaking wave dissipation is represented by shock local energy dissipation [6].

Practically, our numerical investigations have shown that, for a given breaking wave, the switching areas need to be slightly enlarged to prevent the possible occurrence of spurious oscillations. Consequently some neighboring cells are added to the switching area to include the steepening shore facing side of the wave, and a part of the offshore facing side. This can be simply done with the help of a mask, defining the switching area as a band of length $2 r$ centered to the elements verifying $\mathfrak{I}_{j}>1$ (see Fig. 2).

Additionally, the use of a local limitation procedure $\mathbf{w}_{\mathfrak{h}} \leftarrow \Pi_{\mathfrak{h}}$ is classically required in troubled elements. In this work, we use the improved moment limiting strategy of [8]. The reader is referred to [19] for the detailed implementation of this limiting strategy in our NSW equations based numerical model.

\subsection{Robustness}

An additional limitation is performed to ensure the positivity of the water height in the vicinity of dry (or almost dry) areas during the computation. Let us consider $h_{\mathfrak{h} \mid \mathcal{E}^{j}}^{n}(x)$ obtained at the discrete time $t^{n}$ from the fully discrete previous dG method (i.e. deduced from $\eta_{\mathfrak{h} \mid \mathcal{E}^{j}}^{n}(x)$ and $z_{\mathfrak{h} \mid \mathcal{E}^{j}}(x)$ ) and let denote $\bar{h}_{j}^{n}$ its average. Practically, starting from positive mean values $\bar{h}_{j}^{n} \geq 0$ on all element $\left\{\mathcal{E}^{j}\right\}_{1, \ldots, N_{e}}$, we want to ensure that $\bar{h}_{j}^{n+1} \geq 0$ on all element $\left\{\mathcal{E}^{j}\right\}_{1, . ., N_{e}}$, without destroying the order of accuracy.

Such a property is enforced adapting the ideas developed in $[79,81]$ to the pre-balanced formulation. We first note that the scheme satisfied by the cell averaged of the free surface in the dG approximation (28) is

$$
\bar{\eta}_{j}^{n+1}=\bar{\eta}_{j}^{n}-\frac{\Delta_{t}^{n}}{\left|\mathcal{E}^{j}\right|}[\widehat{q}]_{x_{l}^{j}}^{x^{j}}
$$


with $\bar{\eta}_{j}^{n}=\frac{1}{\left|\mathcal{E}^{j}\right|} \int_{\mathcal{E}^{j}} \eta_{\mathfrak{h}}\left(x, t^{n}\right) d x$ and the interface fluxes $\widehat{q}$ given by (46). In the framework of the pre-balanced formulation, as we work with $\eta$ instead of $h$, we have to ensure that the first order scheme associated with (53) preserve the positivity of the water height. To this purpose, let us adapt the definitions of the interfaces interior and exterior values (41)-(42) to the discrete first order case, using piecewise constant data:

$$
\begin{aligned}
& \mathbf{w}_{r}^{-}=\mathbf{w}_{l}^{-}=\mathbf{w}_{j}^{n}, \quad z_{r}^{-}=z_{l}^{-}=z_{j}, \\
& \mathbf{w}_{r}^{+}=\mathbf{w}_{j+1}^{n}, \quad z_{r}^{+}=z_{j+1} \quad \text { and } \quad \mathbf{w}_{l}^{+}=\mathbf{w}_{j-1}^{n}, \quad z_{l}^{+}=z_{j-1} .
\end{aligned}
$$

Proposition 2.10. We consider the following first-order scheme

$$
\eta_{j}^{n+1}=\eta_{j}^{n}-\frac{\Delta_{t}^{n}}{\left|\mathcal{E}^{j}\right|}\left(F_{\eta}\left(\breve{\mathbf{w}}_{r}^{-}, \breve{\mathbf{w}}_{r}^{+}\right)-F_{\eta}\left(\breve{\mathbf{w}}_{l}^{-}, \breve{\mathbf{w}}_{l}^{+}\right)\right)
$$

with $\breve{\mathbf{w}}_{r}^{ \pm}$and $\breve{\mathbf{w}}_{l}^{ \pm}$defined at each interfaces following (43)-(44)-(45) and (54)-(55). If $\eta_{j}^{n}-z_{j}$ and $\eta_{j \pm 1}^{n}-z_{j \pm 1}$ are positive, then $\eta_{j}^{n+1}-z_{j}$ is positive under the condition $\frac{\Delta_{t}^{n}}{\left|\mathcal{E}^{j}\right|} \alpha_{0} \leq 1$.

Proof. The scheme (56) can be written, using (48):

$$
\eta_{j}^{n+1}=\eta_{j}^{n}-\frac{\Delta_{t}^{n}}{2\left|\mathcal{E}^{j}\right|}\left(\left(\frac{\breve{h}_{r}^{-}}{h_{j}^{n}} q_{j}^{n}+\frac{\breve{h}_{r}^{+}}{h_{j+1}^{n}} q_{j+1}^{n}\right)-\alpha_{0}\left(\breve{\eta}_{r}^{+}-\breve{\eta}_{r}^{-}\right)-\left(\frac{\breve{h}_{l}^{-}}{h_{j}^{n}} q_{j}^{n}+\frac{\breve{h}_{l}^{+}}{h_{j-1}^{n}} q_{j-1}^{n}\right)+\alpha_{0}\left(\breve{\eta}_{l}^{-}-\breve{\eta}_{l}^{+}\right)\right)
$$

where we set $h_{j}^{n}=\eta_{j}^{n}-z_{j}$ for the sake of simplicity. Denoting that $\breve{\eta}_{r}^{+}-\breve{\eta}_{r}^{-}=\breve{h}_{r}^{+}-\breve{h}_{r}^{-}$and $\breve{\eta}_{l}^{-}-\breve{\eta}_{l}^{+}=\breve{h}_{l}^{-}-\breve{h}_{l}^{+}$, and subtracting $z_{j}$ at both sides, we can conclude as in [79], writing (56) as a linear combination of positive values:

$$
\begin{aligned}
\eta_{j}^{n+1}-z_{j}=(1- & \left.\frac{\Delta_{t}^{n}}{2\left|\mathcal{E}^{j}\right|}\left(\alpha_{0}+\frac{q_{j}^{n}}{h_{j}^{n}}\right) \frac{\breve{h}_{r}^{-}}{h_{j}^{n}}--\frac{\Delta_{t}^{n}}{2\left|\mathcal{E}^{j}\right|}\left(\alpha_{0}-\frac{q_{j}^{n}}{h_{j}^{n}}\right) \frac{\breve{h}_{l}^{-}}{h_{j}^{n}}\right) h_{j}^{n} \\
& +\left(\frac{\Delta_{t}^{n}}{2\left|\mathcal{E}^{j}\right|}\left(\alpha_{0}+\frac{q_{j-1}^{n}}{h_{j-1}^{n}}\right) \frac{\breve{h}_{l}^{+}}{h_{j-1}^{n}}\right) h_{j-1}^{n}+\left(\frac{\Delta_{t}^{n}}{2\left|\mathcal{E}^{j}\right|}\left(\alpha_{0}-\frac{q_{j+1}^{n}}{h_{j+1}^{n}}\right) \frac{\breve{h}_{r}^{+}}{h_{j+1}^{n}}\right) h_{j+1}^{n} .
\end{aligned}
$$

Based on Proposition 2.10, the ideas of [79] can be directly applied. Let us briefly recall the main steps of the procedure for a $1^{s t}$ order time discretization. For each element $\mathcal{E}^{j}$, assuming that $\bar{h}_{j}^{n} \geq 0$ :

(1) let $S_{i}^{j}=\left\{r_{k}^{j}\right\}_{k=1, . ., d}$ be the set of $d$ LGL points on the element $\mathcal{E}^{j}$, and $\left\{\hat{\omega}_{k}\right\}_{k=1, . ., d}$ the corresponding quadrature weights. $d$ is chosen such that the associated quadrature rule is exact for polynomials of degree $N$ (i.e. $2 d-3 \geq N$ ). We compute $m_{j}^{n}=\min _{r_{k}^{j} \in S_{i}^{j}} h_{\mathfrak{h} \mid \mathcal{E}^{j}}^{n}\left(r_{k}^{j}\right)$.

(2) we modify $h_{\mathfrak{h} \mid \mathcal{E}^{j}}^{n}(x)$ in order to ensure that it is positive at the previous set of $d$ LGL nodes. This is done using the following conservative accuracy-preserving linear scaling around the cell average:

$$
\check{h}_{\mathfrak{h} \mid \mathcal{E}^{j}}^{n}(x)=\theta_{j}^{n}\left(h_{\mathfrak{h} \mid \mathcal{E}^{j}}^{n}(x)-\bar{h}_{j}^{n}\right)+\bar{h}_{j}^{n} .
$$

where

$$
\theta_{j}^{n}=\min \left(\frac{\bar{h}_{j}^{n}}{\bar{h}_{j}^{n}-m_{j}^{n}}, 1\right) .
$$

We deduce from (58) a modified $N$ order polynomial $\check{\eta}_{\mathfrak{h} \mid \mathcal{E}^{j}}^{n}(x)$, which is injected into (28)-(29) instead of (27). 
Thus, following $[79,81]$, the positivity of the mean water height $\bar{h}_{j}^{n+1}$ is ensured under the condition :

$$
\alpha_{0} \frac{\Delta_{t}^{n}}{\left|\mathcal{E}^{j}\right|} \leq \hat{w}_{1}
$$

In practice, we have $\hat{w}_{1}=\frac{1}{6}$ for $N=2,3$ and $\frac{1}{12}$ for $N=4,5$.

\subsection{Boundary conditions}

The boundary conditions are classically imposed weakly, by enforcing suitable reflecting relations at a virtual exterior nodes, at each boundaries, through the boundary interface fluxes. Periodic, Neumann and Dirichlet conditions can be enforced following this simple process. For the approximation of second order derivatives with the LDG strategy, we classically enforce Neumann boundary conditions for the auxiliary variable. We also use Neumann boundary conditions for the derivatives involved in the discrete version of the operator $1+\alpha \mathfrak{T}\left[h_{b}\right]$. The first and last rows of (36) should be adapted for Dirichlet conditions. As far as generation and absorption of waves are concerned, we use relaxation techniques, as in [9,43]. The computational domain is slightly extended to include the sponge layers, which length has to be calibrated from the incoming waves (generally 2 wavelengths). We enforce periodic waves generation with the use of a generation/relaxation zone, following [50]. We have implemented the relaxation functions proposed in [75] and at the inlet boundary, we progressively impose on a two-wavelength long generation layer the targeted wave train.

\section{Numerical EXAMPLES}

In this section, we assess the ability of our $\mathrm{dG}$ approach to compute waves propagation and transformations. The two models/schemes given by (28)-(29) involving (21a) or (21b) will be referred to as GNO (for original) and GNC (for constant) respectively. Unless stated otherwise, we use Neumann boundary conditions at both boundaries, the optimized value $\alpha=1.159$ (see [7]) is used and we set $\epsilon_{b}=0.1$. We highlight that our numerical investigations have shown that the value of $\epsilon_{b}$ does not influence the numerical results. Unless stated otherwise, and accordingly with the robustness result of the previous section, we do not suppress the dispersive effects in the vicinity of dry areas.

\subsection{Motionless steady states preservation}

We consider a $100 \mathrm{~m}$ channel and start from an initial steady state at rest $\eta=0$ and $q=0$, over the following topography (see Fig. 3):

$$
z(x)=-h_{0} \exp \left(-((x-d) / l)^{2}\right)+0.8 .
$$

After $10 s$ of simulation, the steady state is still preserved up to the machine accuracy, regardless of the polynomials' order in the expansion basis or the refinement of the mesh. As an example, for $N=3$ and $N_{e}=300$, the computations give an $L^{1}$-error of $1.56 e^{-16}$ for the free surface and $7.13 e^{-15}$ for the discharge.

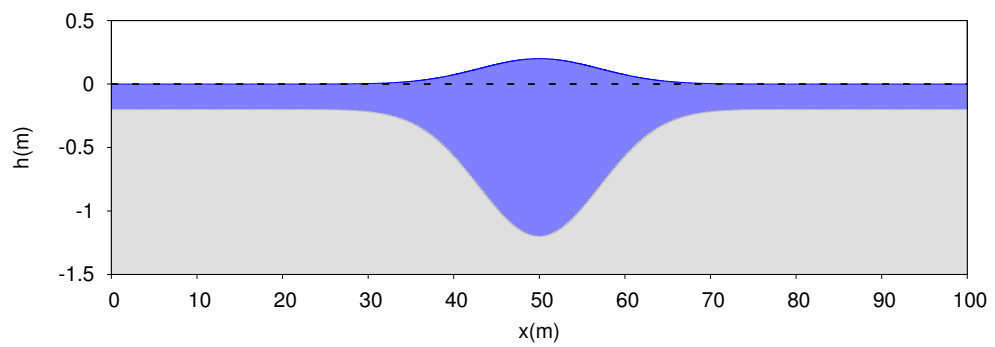

Figure 3. Topography and initial conditions for test cases 3.1 and 3.2. 


\subsection{Accuracy and convergence analysis in the presence of non-flat bottom}

We now focus on the evolution of a smooth gaussian profile over the previous non flat and smooth topography, to highlight the convergence properties of our approach. The initial water height is defined as $h(x)=a z(x)$, where $a / h_{0}=0.2$ and $z$ defined in the previous test case (59). No exact solution is available, so a reference solution is computed at $t=0.15 \mathrm{~s}$ with $N=7, N_{e}=1280$. Computations for both models (20)-(21a) and (20)-(21b) are run on a sequence of regular meshes with increasing refinement and polynomial expansions of increasing orders, from 1 to 5 . For the approximation of high order derivatives, we use successively LDG and BR fluxes. The numerical $L^{1}$-errors computed using the reference solution at $t=0.15 \mathrm{~s}$ are reported in Tab. 5 for the water height and plotted on Fig. 4 for both water height and discharge, in log-log scale.
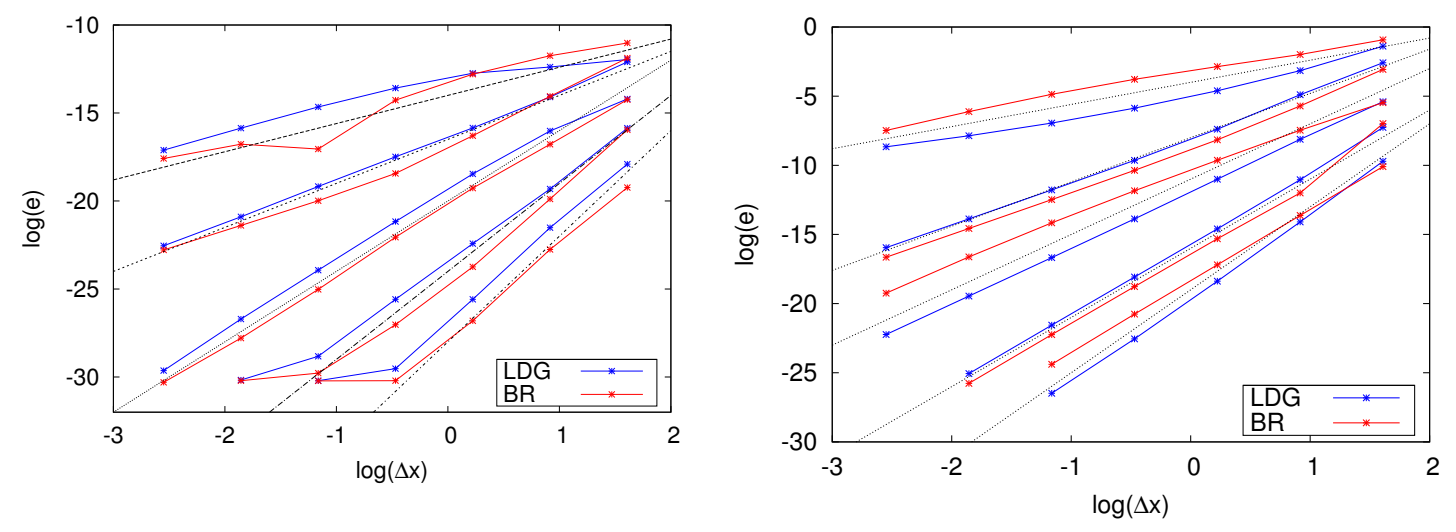

FIGURE 4. Accuracy analysis in the presence of non-flat bottom: convergence rates for the water height (left) and the discharge (right) - reference slopes 1.6, 3.2, 4, 5 and 6 are plotted in dotted lines

As expected, we obtain similar orders of accuracy for both GNO and GNC models. Concerning the impact of the numerical flux choice on the convergence rates, we can observe on Table (5) that, for both models and as mentioned in previous studies $[15,22,30,31]$, the use of BR fluxes may lead to sub-optimal convergence rates for odd values of $N$, while the LDG fluxes lead to optimal convergence rates. The corresponding convergence orders are reported on the last column of Table (5).

Let us now investigate the computational improvements obtained with the new GNC model. To this purpose, an averaged cpu-time per time-step is measured for both models, for increasing values of polynomials' order and number of elements. These cpu times are denoted respectively $\rho_{o}$ and $\rho_{c}$ for the original and constant models. We focus on the ratio $\tau:=\rho_{o} / \rho_{c}$ and the corresponding values are reported in Tab. 6. These values confirm the computational savings provided by the GNC model, clearly more efficient for all given couple $\left(N, N_{e}\right)$. We observe that the ratio increases with respect to the polynomial's order, confirming the benefit of this new approach for high order simulations. For polynomial expansions of order 6 the speed-up can reach 7.15. 


\begin{tabular}{|c|c|c|c|c|c|c|c|c|c|}
\hline Model & Fluxes & $N$ & 20 & 40 & 80 & $\begin{array}{c}N_{e} \\
160\end{array}$ & 320 & 640 & order \\
\hline \multirow[t]{10}{*}{ GNO } & BR & 1 & 0.4 & 0.13 & $5.6 \mathrm{e}-2$ & $2.2 \mathrm{e}-2$ & $7.6 \mathrm{e}-3$ & $2.2 \mathrm{e}-3$ & 1.5 \\
\hline & & 2 & $4.4 \mathrm{e}-2$ & $3.2 \mathrm{e}-3$ & $2.9 \mathrm{e}-4$ & $3.2 \mathrm{e}-5$ & $3.9 \mathrm{e}-6$ & $4.9 \mathrm{e}-7$ & 3.3 \\
\hline & & 3 & $4.1 \mathrm{e}-3$ & $5.4 \mathrm{e}-4$ & $6.1 \mathrm{e}-5$ & $6.7 \mathrm{e}-6$ & $6.6 \mathrm{e}-7$ & $5.6 \mathrm{e}-8$ & 3.2 \\
\hline & & 4 & $7.7 \mathrm{e}-4$ & $6.1 \mathrm{e}-6$ & $2.2 \mathrm{e}-7$ & $6.9 \mathrm{e}-9$ & $2.1 \mathrm{e}-10$ & $6.7 \mathrm{e}-12$ & 5.3 \\
\hline & & 5 & $3.6 \mathrm{e}-5$ & $1.1 \mathrm{e}-6$ & $3.1 \mathrm{e}-8$ & $8.7 e-10$ & $2.3 e-11$ & / & 5.1 \\
\hline & LDG & 1 & 0.25 & $4.3 \mathrm{e}-2$ & $1.0 \mathrm{e}-3$ & $2.9 \mathrm{e}-3$ & $9.7 \mathrm{e}-4$ & $3.8 \mathrm{e}-4$ & 1.9 \\
\hline & & 2 & $7.3 \mathrm{e}-2$ & $7.3 \mathrm{e}-3$ & $6.2 \mathrm{e}-4$ & $6.6 e-5$ & $7.9 \mathrm{e}-6$ & $9.8 \mathrm{e}-7$ & 3.2 \\
\hline & & 3 & $4.3 \mathrm{e}-3$ & $2.9 \mathrm{e}-4$ & $1.6 \mathrm{e}-5$ & $9.0 \mathrm{e}-7$ & $5.5 \mathrm{e}-8$ & $3.4 \mathrm{e}-9$ & 4.0 \\
\hline & & 4 & $6.6 \mathrm{e}-4$ & $1.5 \mathrm{e}-5$ & $4.4 \mathrm{e}-7$ & $1.4 \mathrm{e}-8$ & $4.2 \mathrm{e}-10$ & $1.3 \mathrm{e}-11$ & 5.1 \\
\hline & & 5 & $5.2 \mathrm{e}-5$ & $7.0 \mathrm{e}-7$ & $9.6 \mathrm{e}-9$ & $1.5 \mathrm{e}-10$ & $2.8 \mathrm{e}-12$ & / & 6.0 \\
\hline \multirow[t]{10}{*}{ GNC } & BR & 1 & 0.4 & 0.14 & $5.7 \mathrm{e}-2$ & $2.3 e-2$ & $7.7 \mathrm{e}-3$ & $2.2 \mathrm{e}-3$ & 1.5 \\
\hline & & 2 & $4.7 \mathrm{e}-2$ & $3.3 e-3$ & $2.9 \mathrm{e}-4$ & $3.2 \mathrm{e}-5$ & $3.8 \mathrm{e}-6$ & $4.7 \mathrm{e}-7$ & 3.3 \\
\hline & & 3 & $4.2 \mathrm{e}-3$ & $5.8 \mathrm{e}-4$ & $6.5 \mathrm{e}-5$ & $7.2 \mathrm{e}-6$ & $7.1 \mathrm{e}-7$ & $6.0 \mathrm{e}-8$ & 3.2 \\
\hline & & 4 & $9.3 \mathrm{e}-4$ & $6.2 \mathrm{e}-6$ & $2.2 \mathrm{e}-7$ & $7.0 \mathrm{e}-9$ & $2.2 \mathrm{e}-10$ & $6.4 \mathrm{e}-12$ & 5.4 \\
\hline & & 5 & $4.1 \mathrm{e}-5$ & $1.2 \mathrm{e}-6$ & $3.4 \mathrm{e}-8$ & $9.6 \mathrm{e}-10$ & $2.6 \mathrm{e}-11$ & / & 5.1 \\
\hline & $\mathrm{LDG}$ & 1 & 0.25 & $4.2 \mathrm{e}-2$ & $1.0 \mathrm{e}-3$ & $2.8 \mathrm{e}-3$ & $9.6 \mathrm{e}-4$ & $3.9 \mathrm{e}-4$ & 1.9 \\
\hline & & 2 & $7.5 \mathrm{e}-2$ & $7.5 \mathrm{e}-3$ & $6.2 \mathrm{e}-4$ & $6.5 \mathrm{e}-5$ & $7.7 \mathrm{e}-6$ & $9.5 \mathrm{e}-7$ & 3.2 \\
\hline & & 3 & $4.5 \mathrm{e}-3$ & $3.0 \mathrm{e}-4$ & $1.7 e-5$ & $9.4 \mathrm{e}-7$ & $5.7 \mathrm{e}-8$ & $3.6 e-9$ & 4.0 \\
\hline & & 4 & $7.0 \mathrm{e}-4$ & $1.6 e-5$ & $4.6 \mathrm{e}-7$ & $1.4 \mathrm{e}-8$ & $4.4 \mathrm{e}-10$ & $1.3 \mathrm{e}-11$ & 5.1 \\
\hline & & 5 & $6.1 \mathrm{e}-5$ & $7.6 \mathrm{e}-7$ & $1.0 \mathrm{e}-8$ & $1.6 \mathrm{e}-10$ & $3.1 \mathrm{e}-12$ & / & 6.1 \\
\hline
\end{tabular}

Figure 5. Accuracy analysis in the presence of non-flat bottom: $L^{1}$ error for the discharge.

\begin{tabular}{|c|c|c|c|c|c|c|}
\hline & \multicolumn{6}{|c|}{$N_{e}$} \\
$N$ & 1000 & 2000 & 3000 & 4000 & 5000 & 6000 \\
\hline \hline 1 & 3.23 & 3.21 & 3.04 & 2.96 & 2.94 & 2.90 \\
2 & 4.09 & 4.27 & 4.14 & 4.01 & 3.90 & 3.84 \\
3 & 5.32 & 5.11 & 5.03 & 4.97 & 4.91 & 4.87 \\
4 & 6.01 & 5.77 & 5.67 & 5.63 & 5.51 & 5.55 \\
5 & 6.66 & 6.38 & 6.32 & 6.30 & 6.26 & 6.16 \\
6 & 7.15 & 6.99 & 7.05 & 6.97 & 6.86 & 6.54 \\
\hline
\end{tabular}

FiguRE 6. Accuracy analysis in the presence of non-flat bottom: time ratio $\tau=\rho_{o} / \rho_{c}$ for increasing values of $N$ and $N_{e}$.

Based on these investigations, all the following computations are performed with the GNC model.

\subsection{Propagation of a solitary wave}

In this test, we investigate the propagation of a solitary wave over a flat bottom. We recall that the original model (1) admits the following class of solitary waves solution:

$$
\left\{\begin{array}{l}
h(x, t)=h_{0}+a \operatorname{sech}^{2}(\kappa(x-c t)) \\
u(x, t)=c\left(1-\frac{h_{0}}{h(x, t)}\right)
\end{array}\right.
$$

where $\kappa=\sqrt{\frac{3 a}{4 h_{0}^{2}\left(h_{0}+a\right)}}$, and $c=\sqrt{g\left(h_{0}+a\right)}$. We also recall that these solitary waves are only solutions up to $O\left(\mu^{2}\right)$ of the new GNC family of models. 


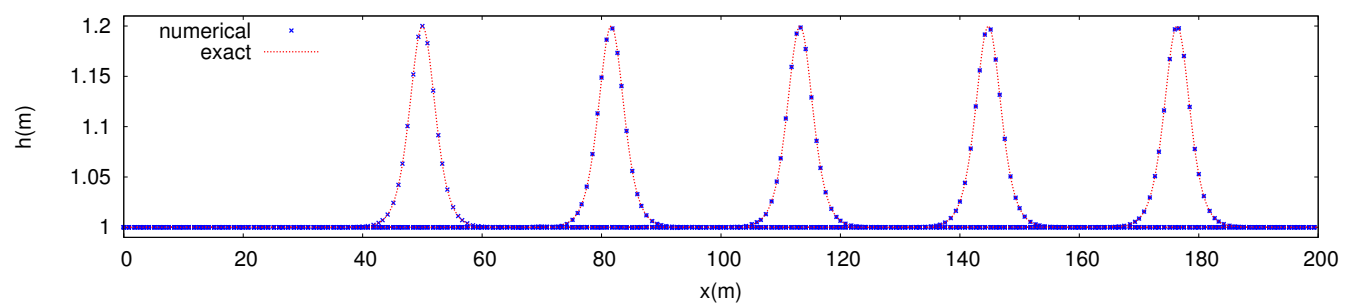

Figure 7. Propagation of a solitary wave : water surface profiles at $\mathrm{t}=0.4,0.8,1.2,1.6$ and $2 \mathrm{~s}$.

For this test, the reference water height is fixed at $h_{0}=1 \mathrm{~m}$ and we use the new model (12), with $\alpha=1$ to compute the propagation of a solitary wave initially centered at $x_{0}=50 \mathrm{~m}$, with a relative amplitude set to $a=0.2 h_{0}$. The computational domain is a $200 \mathrm{~m}$ long channel, regularly meshed with 400 elements. We investigate several orders of polynomial expansions and follow the flow motion along the channel until $t=2.2 \mathrm{~s}$. We show on (3.3) some free surface profiles at several times obtained with $3^{\text {rd }}$ order expansions and a fourth order SSP-RK time-marching scheme. We can observe an excellent agreement between numerical solutions provided by the new model (12) and formula (60), showing the negligible discrepancies introduced by the $O\left(\mu^{2}\right)$ approximation in the new family of models. The free surface profile is accurately preserved during the propagation. However, let us mention that even if we do not report results here, some convergence studies have been performed for this particular test case, and we obtain sub-optimal $N+\frac{1}{2}$ convergence rates, for both BR and LDG fluxes. The propagation and transformations of solitary waves of increased non-linearity are studied in the next test.
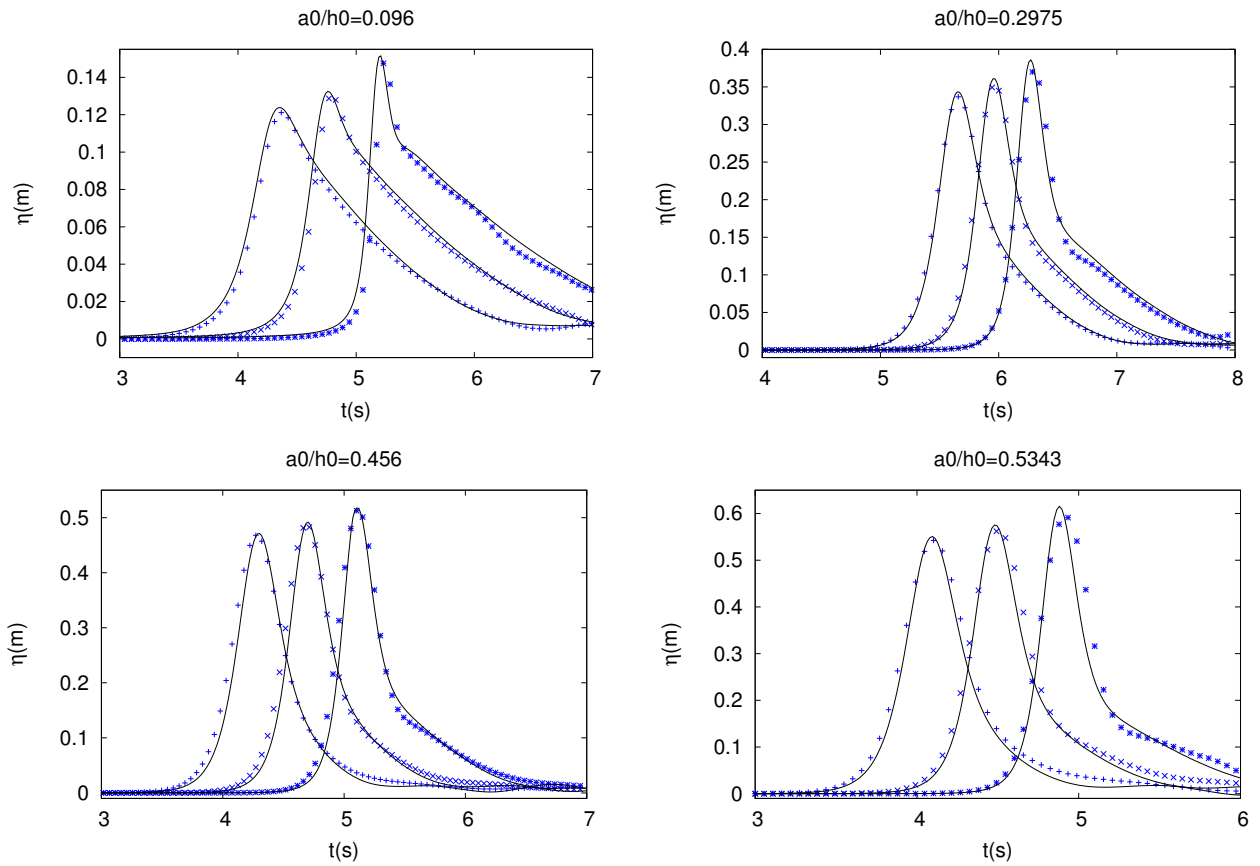

FiguRE 8. Guibourg test case : comparison between computed (solid lines) and experimental (dots) time series of total free surface at several gauges before the breaking point. 


\subsection{Shoaling of solitary waves ( [35])}

We now investigate the dispersive properties of the model and study the nonlinear shoaling, using the data issued from a laboratory study performed at the LEGI (Grenoble, France). In this test, we consider a $36 \mathrm{~m}$ channel with constant bed slope and a train of solitary waves generated at the inflow boundary. Measurements of the free surface are available at several wave gages in the vicinity of the breaking point during the simulation. We consider 4 series of experiments, involving an increasing relative amplitude, starting from $a / h_{0}=0.096$ and ending with $a / h_{0}=0.534$.

The displayed numerical results are obtained with $\left(N, N_{e}\right)=(3,400)$. Numerical results are reported on Fig. 8, together with the experimental data available from [35]. We can observe a very good behavior of the numerical model, and even for large wave's amplitude. These results assess the good accuracy of our model in the reproduction of the shoaling process.

\subsection{Periodic waves over a submerged bar ( [18])}

Going ahead in the assessment of the dispersive properties of the GNC model, we investigate now the propagation of periodic waves over a submerged bar, following the set-up proposed in [18]. The topography set-up and wave gauges locations are shown in Fig. 9. We choose here to perform test A, in which the amplitude of the input waves is set to $a=0.01 \mathrm{~m}$. The time period $T$ is $2.02 \mathrm{~s}$ and the initial depth $h_{0}$ is $0.4 m$. When the incident waves encounters the upward part of the bar, it shoals and steepens, which generates higherharmonics as the nonlinearity increases. These higher-harmonics are then freely released on the downward slope, and become deep-water waves behind the bar. The domain is regularly meshed with $N_{e}=800$ elements, and computations are run with increasing orders of polynomial expansions. Incident waves do not encounter breaking during the propagation, so that the switching/limiting process is not activated in this test.

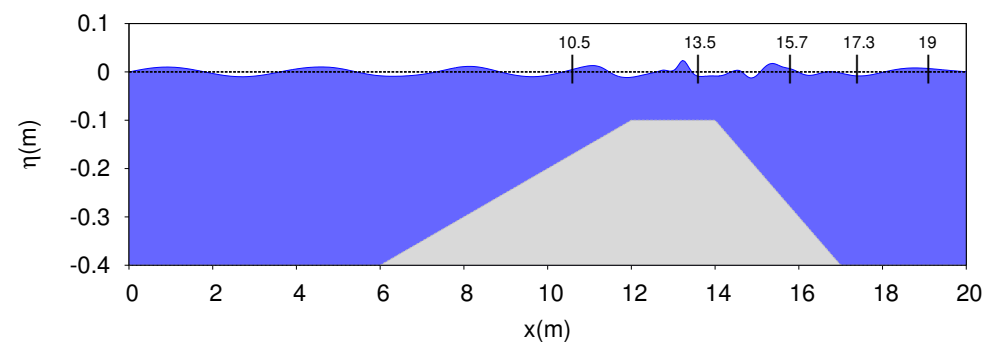

Figure 9. Periodic waves over a submerged bar : sketch of the basin and gauges location.

We show on Fig. 10 some time series of the free surface elevation at several wave gauges located along the channel, compared with the experimental data. These results are obtained with $3^{\text {rd }}$ order polynomials. We obtain a very good agreement at the first gauges. Some discrepancies are noticed at the last gauge, mostly explained by the high non-linear interactions generated as the waves encounters the upward part of the bar. As exhibited in Fig. 11, these effects are not correctly reproduced up to $N=3$ at the last gauge for this choice of space step and we tried to locally increase the model accuracy, leading to sensible improvements. However, in the area of the last three gauges, the dispersion properties of the current model are no more satisfactory enough to accurately reproduce the complete release of the higher-harmonics, which can be regarded as highly dispersive waves. These results can be improved with the use of some optimized GN models, as for instance the 3 parameters optimized Green-Naghdi models proposed in [9] and in the 2d case in [43]. The dG approximations of such enhanced GN equations are left for future works. 

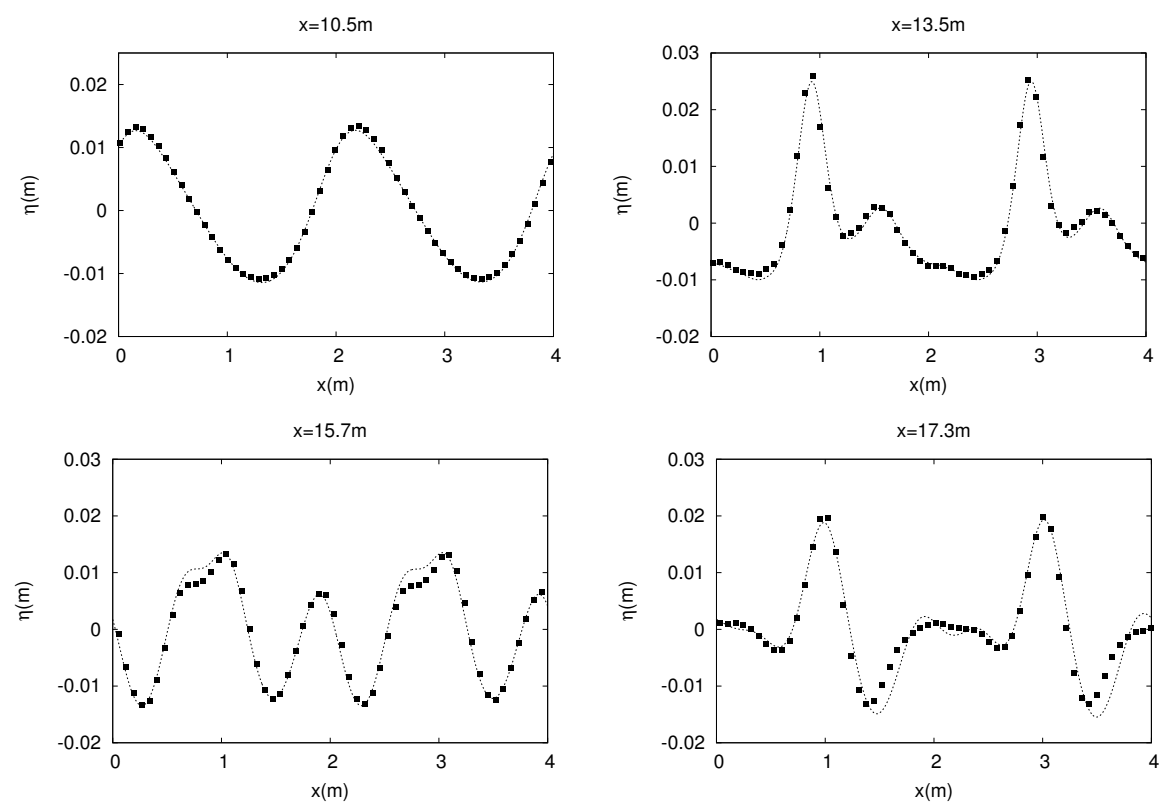

FiguRe 10. Propagation of highly dispersive waves : free surface evolution at the four first gauges. Experimental data are denoted by squares.

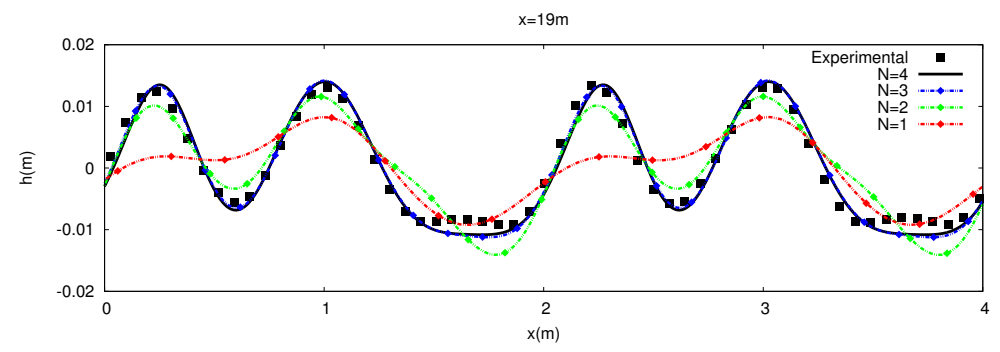

Figure 11. Propagation of highly dispersive waves : several profiles for increasing $N$ at the last gauge.

\subsection{Solitary wave breaking over a sloping beach ( [67])}

Let us now assess the ability of our numerical model to deal with breaking waves and dry areas. In this test, we study the propagation, shoaling, breaking and run-up processes of a solitary wave over a beach with constant slope $s=1 / 19.85$, following the experiments of [67]. The incident wave is supplied by formula (60), with a water level at rest $h_{0}=1 \mathrm{~m}$ and an amplitude set to $a=0.28 \mathrm{~m}$. The couple $\left(N, N_{e}\right)$ is fixed to $(2,800)$ for the computation and our numerical results are compared with the experimental data. We observe that the breaking wave method described in ( $\$ 2.6)$ is able to identify the wave steepening, occurring approximatively around $t^{*}=17 \mathrm{~s}$. We emphasize the very satisfying agreement between the numerical results and the data, as shown on Fig. 12. 

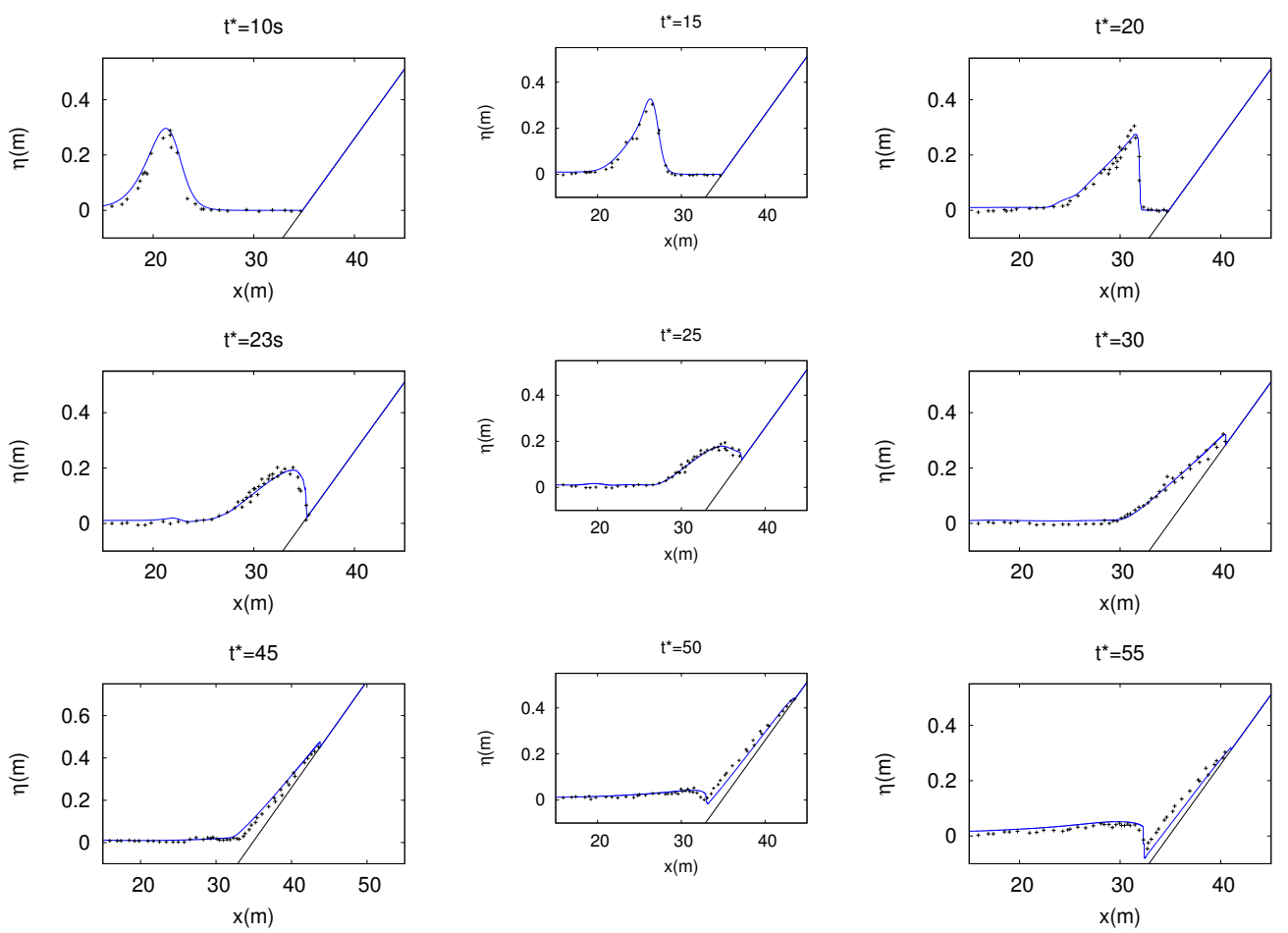

FIGURE 12. Solitary wave breaking over a sloping beach : free surface profiles comparison between numerical results (solid lines) and experimental data (dots) at several times during the breaking; $t^{*}=t\left(g / h_{0}\right)^{1 / 2}$.

\subsection{Cox's experiment ( $[16])$}

To further investigate the breaking waves / switching strategy, we study in this test the evolution of an incoming train of regular waves over a beach with constant bed slope $1 / 35$. Waves of relative amplitude $a / h_{0}=0.29$ and period $T=2.2 \mathrm{~s}$ are generated at the inflow boundary. We set $N=1$ and use two mesh sizes for this test: $\left|\mathcal{E}_{j}\right|=0.0575$ for $x<11.5 \mathrm{~m}$ and $\left|\mathcal{E}_{j}\right|=0.028$ for $x \geq 11.5 \mathrm{~m}$. A sketch of the computational domain is available in Fig. 13, with the location of the wave gauges.

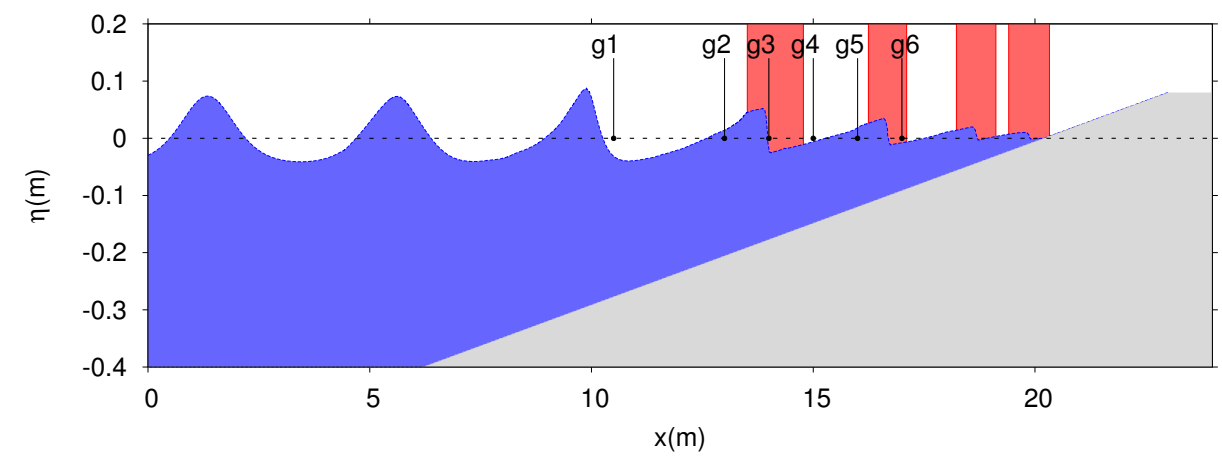

FiguRE 13. Cox's experiment : sketch of the basin and identification of breaking areas. The NSW equations are used in red areas to describe the breaking waves. 
In this picture, we also point out the areas where the dispersive effects are turned off (in red) and the NSW equations are used, following the switching strategy detailed in §2.6. In agreement with experimental observations, the first wave to break is detected between gauges $\sharp 2$ and $\sharp 3$. Note that, for this particular test case, the dispersive terms are switched off in the vicinity of the shoreline because the switching criteria $\mathfrak{I}$ is larger than 1 in this area.
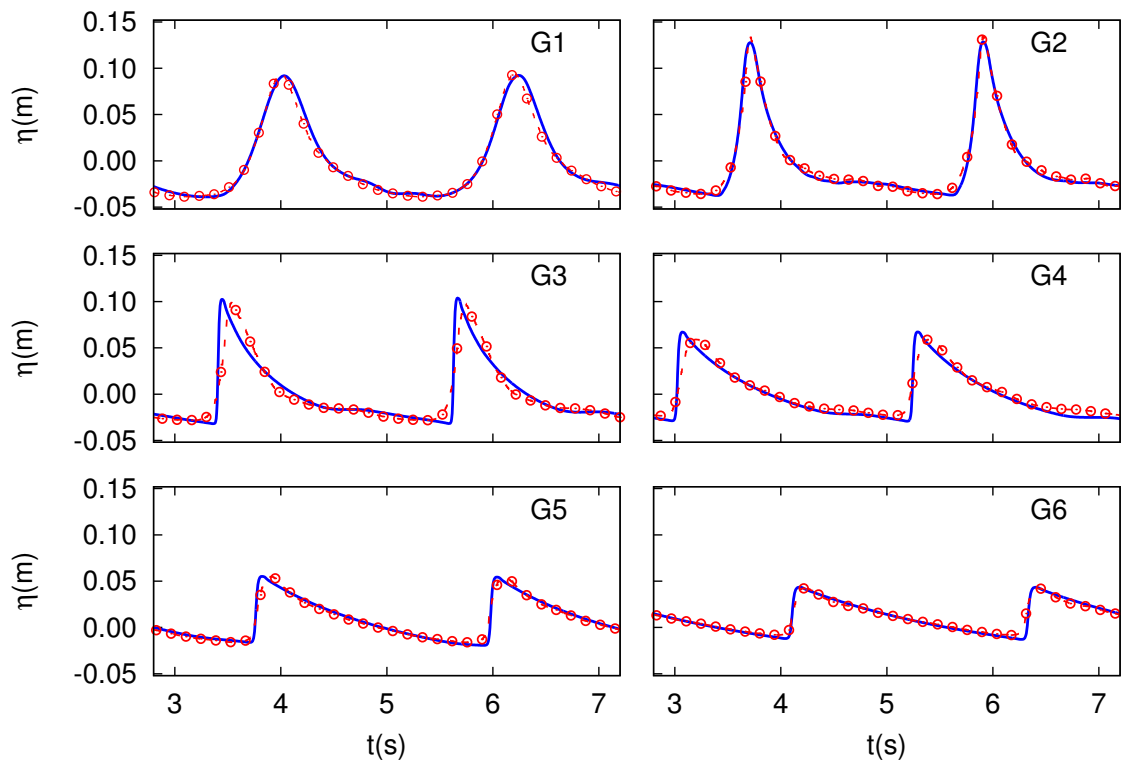

FiguRE 14. Cox's experiment : comparison between computed and experimental time series of total free surface at the gauges.

We can observe on Fig. 14 the time series of the free surface elevation at the six wave gauges along the domain and the comparison with the data taken from the experiment. We observe a very good matching. A similar level of agreement can be obtained by reducing the number of elements and increasing the polynomials order, for instance $N=2$ and $\left|\mathcal{E}_{j}\right|=0.1$. Note also that the limiter [8] is not applied in the whole switching (red) areas but only on the few troubled elements in the vicinity of the discontinuities.

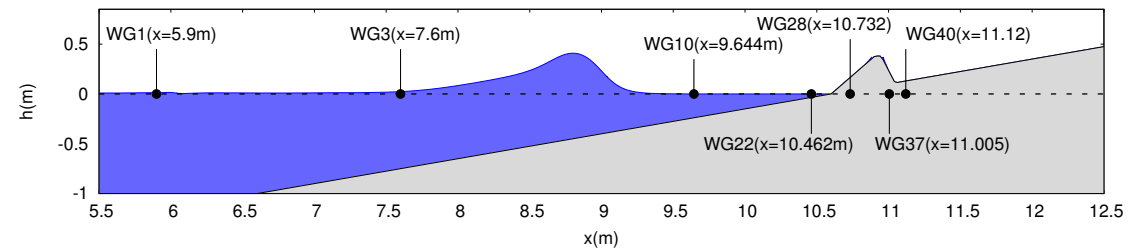

FiguRE 15. Overtopping over fringing reefs : sketch of the basin and gauge locations. 


\subsection{Wave overtopping a seawall ( [36])}

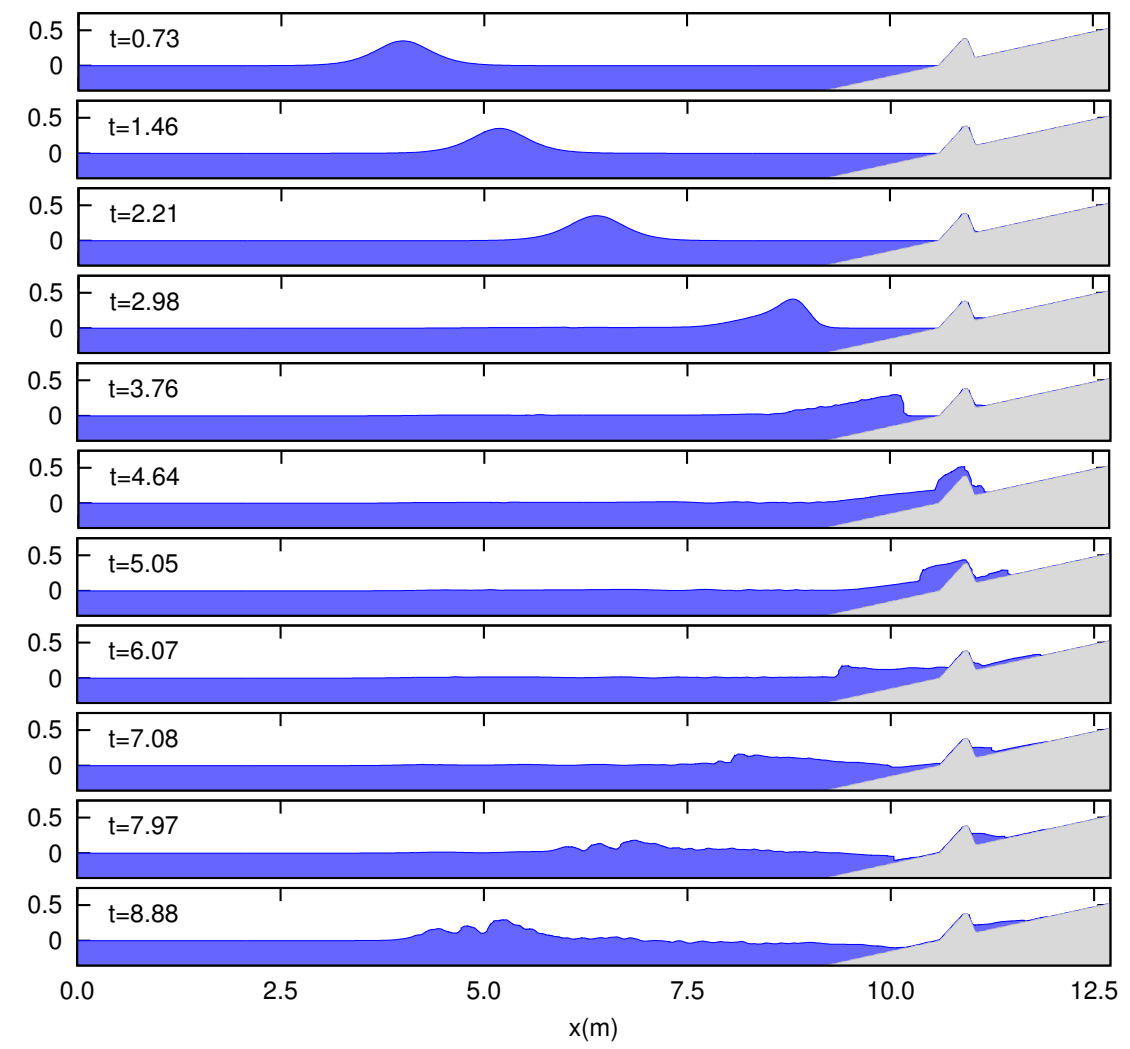

FIGURE 16. Overtopping over fringing reefs : Free surface profiles at several times during the propagation.

In this last validation, we aim at demonstrating that our numerical model appear as a promising tool for more complex simulations. The following test is based on the experiments carried on at the Tainan Hydraulics Laboratory (THL), National Cheng Kung University. It implies a tsunami-like solitary wave collapsing on a seawall located on a 1:20 sloping beach. A cross-section of the $22 \mathrm{~m}$ flume is described in Fig. 15, together with the location of several gauges where measurements of the free surface are available (see [36] for more details). We consider an initial water depth of $0.2 \mathrm{~m}$, and an incoming wave with a relative amplitude $a / h_{0}=0.35$. For this simulation we set $N=2$ and we use two mesh sizes: $\left|\mathcal{E}_{j}\right|=0.03$ for $9<x<12 \mathrm{~m}$ and $\left|\mathcal{E}_{j}\right|=0.09$ elsewhere, giving a total number of $N_{e}=300$ elements. In this case, the solitary wave encounters breaking before reaching the seawall. It follows an overtopping flow supplemented by a train of reflected waves subsequently generated after the impact on the wall. Time series of the free surface were recorded at several gauges along the computational domain, and are compared with experimental data (see Fig. 17). We can note a very good agreement, similar to those exhibited in [36] with a obtained with a Volume Of Fluid (VOF) method for the Reynolds averaged Navier-Stokes equations and [68] with a hybrid FVM discretization of the original GN equations. Note that a similar level of agreement can be obtained with a regular mesh of 500 elements. 


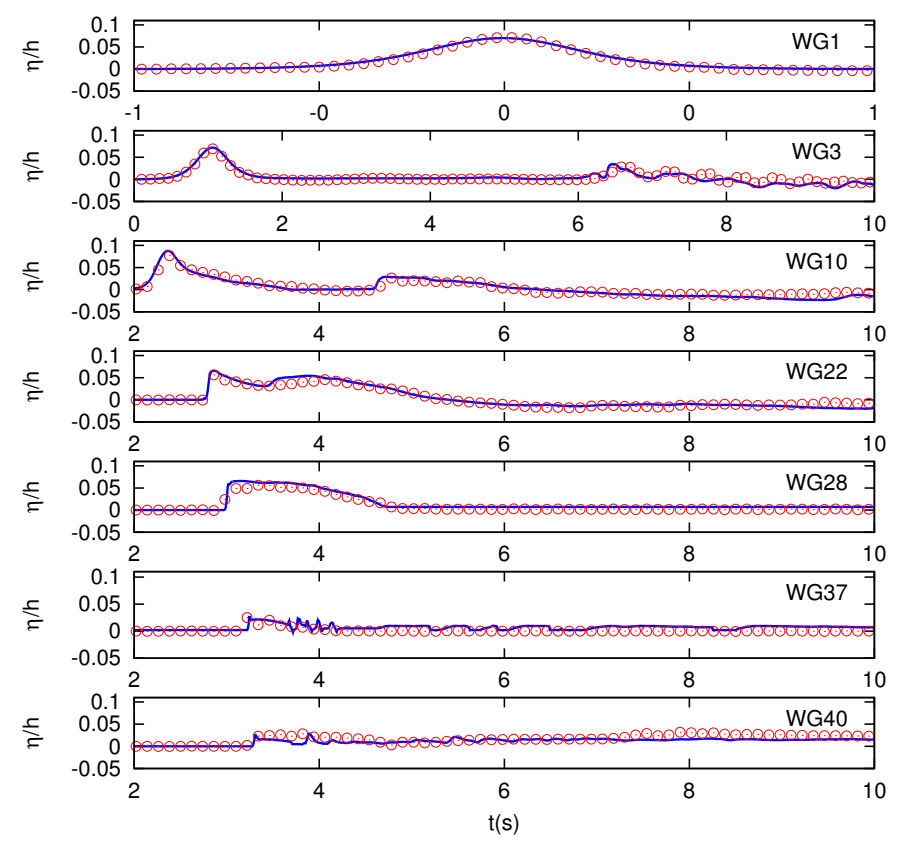

FIGURE 17. Overtopping over fringing reefs : comparison between computed and experimental data of total free surface at several times during the propagation. Circles denote experimental data.

\section{Conclusion}

In this work, an arbitrary order dG discretization is proposed for a new family of 1d Green-Naghdi equations for the simulation of fully non-linear and weakly dispersive waves propagation and transformation over uneven bottom. This approach provides robustness and preservation of motionless steady states. These new models are shown to be more computationally efficient than the original ones, especially for high order polynomial expansions. We also propose a simple way to handle broken wave, which gives satisfying results. The proposed methodology can be straightforwardly extended to the $2 \mathrm{~d}$ framework, with the use of unstructured meshes. The $2 \mathrm{~d}$ models with time-independent operator are already derived in [43] and the extension of the present $\mathrm{dG}$ method is studied in an on-going work. This appears as a promising tool in terms of possible decreasing of the degrees of freedom and local adaptivity in complex geometries.

\section{ACKNOWLEDGMENTS}

The authors would like to thank Prof. S.- C. Hsiao and T.- C. Lin for providing experimental data. The authors would also like to acknowledge additional financial and scientific support of the French INSU-CNRS (Institut National des Sciences de l'Univers-Centre National de la Recherche Scientifique) program LEFEMANU (Méthodes Mathématiques et Numériques), project SOLi. F.M. also acknowledges partial support from the ANR BLANC Bond.

\section{REFERENCES}

[1] B. Alvarez-Samaniego and D. Lannes. A nash-moser theorem for singular evolution equations. application to the serre and green-naghdi equations. Indiana Univ. Math. J., 57:97-131, 2008.

[2] V.R. Ambati and O. Bokhove. Space-time discontinuous Galerkin finite element method for shallow water flows. J. Comput. App. Math., 204:452-462, 2007. 
[3] D.N. Arnold, F. Brezzi, B. Cockburn, and L.D. Marini. Unified analysis of discontinuous Galerkin methods for elliptic problems. SIAM J. Numer. Anal., 39(5):1749-1779, 2002.

[4] F. Bassi and S. Rebay. A high-order accurate discontinuous finite element method for the numerical solution of the compressible Navier-Stokes equations. J. Comput. Phys., 131:267-279, 1997.

[5] C. Berthon and F. Marche. A positive preserving high order VFRoe scheme for shallow water equations: a class of relaxation schemes. SIAM J. Sci. Comput., 30:2587-2612, 2008.

[6] P. Bonneton. Modelling of periodic wave transformation in the inner surf zone. Ocean Engineering, 34(10):1459-1471, 2007.

[7] P. Bonneton, F. Chazel, D. Lannes, F. Marche, and M. Tissier. A splitting approach for the fully nonlinear and weakly dispersive Green - Naghdi model. J. Comput. Phys., 230:1479-1498, 2011.

[8] A. Burbeau, P. Sagaut, and Ch.-H. Bruneau. A problem-independent limiter for high-order runge-kutta discontinuous Galerkin methods. J. Comput. Phys., 169:111-150, 2001.

[9] F. Chazel, D. Lannes, and F. Marche. Numerical simulation of strongly nonlinear and dispersive waves using a Green - Naghdi model. J. Sci. Comput., pages 105-116, 2011.

[10] Q. Chen, J.T. Kirby, R. Dalrymple, and A. Kennedy. Boussinesq modeling of wave transformation, breaking, and runup. II: 2 D. Journal of Waterway, 2000.

[11] R. Cienfuegos, E. Barthélemy, and P. Bonneton. A fourth-order compact finite volume scheme for fully nonlinear and weakly dispersive Boussinesq-type equations. I: Model development and analysis. Internat. J. Numer. Methods Fluids, 51(11):1217$1253,2006$.

[12] R. Cienfuegos, E. Barthelemy, and P. Bonneton. A wave-breaking model for Boussinesq-type equations including mass-induced effects. J. Wtrwy. Port Coast. and Oc. Engrg., 136:10-26, 2010.

[13] R. Cienfuegos, E. Barthélemy, and P. Bonneton. Wave-breaking model for Boussinesq type equations including roller effects in the mass conservation equation. J. of Water. Port. Coast. and Oc. Eng., 136:10-26, 2010.

[14] B. Cockburn and C.-W. Shu. Runge-Kutta discontinuous Galerkin methods for convection-dominated problems. J. Sci. Comput., 16(3):173-260, 2001.

[15] B. Cockburn and S.-W. Shu. The Local Discontinuous Galerkin method for time-dependent convection-diffusion systems. SIAM J. Numer. Anal., 141:2440-2463, 1998.

[16] D.T. Cox. Experimental and numerical modelling of surf zone hydrodynamics. Univ. of Delaware, Newark, 1995.

[17] T.A. Davis and I.S. Duff. An unsymmetric-pattern multifrontal method for sparse LU factorization. SIAM J. on Matrix Anal. and Applications, 18:140-158, 1997.

[18] M.W. Dingemans. Comparison of computations with Boussinesq-like models and laboratory measurements. Delft Hydr., Report H-1684.12, 32, 1994.

[19] A. Duran and F. Marche. Recent advances on the discontinuous Galerkin method for shallow water equations with topography source terms. Submitted, 2013.

[20] A. Duran, F. Marche, and Q. Liang. On the well-balanced numerical discretization of shallow water equations on unstructured meshes. J. Comput. Phys., 235:565-586, 2013.

[21] D. Dutykh, T. Katsaounis, and D. Mitsotakis. Finite volume schemes for dispersive wave propagation and runup. J. Comput. Phys., 230:3035 - 3061, 2011.

[22] A.P. Engsig-Karup, J.S. Hesthaven, H.B. Bingham, and P.A. Madsen. Nodal DG-FEM solution of high-order Boussinesq-type equations. J. Eng. Math., 56:351-370, 2006.

[23] A.P. Engsig-Karup, J.S. Hesthaven, H.B. Bingham, and T. Warburton. DG-FEM solution for nonlinear wave-structure interaction using Boussinesq-type equations. Coast. Eng., 55:197-208, 2008.

[24] K. Erduran, S. Ilic, and V. Kutija. Hybrid finite-volume finite-difference scheme for the solution of Boussinesq equations. Int. J. Numer. Meth. Fluids, 49:1213-1232, 2005.

[25] K.S. Erduran. Further application of hybrid solution to another form of Boussinesq equations and comparisons. Int $J$ Numer Methods Fluids, 53:827-849, 2007.

[26] A. Ern and J.-L. Guermond. Discontinuous Galerkin methods for Friedrichs' systems. i. general theory. SIAM J. Numer. Anal., 44(2):753-778, 2006.

[27] A. Ern and J.-L. Guermond. Discontinuous Galerkin methods for Friedrichs' systems. ii. second-order elliptic pdes. SIAM J. Numer. Anal., 44(6):2363-2388, 2006.

[28] A. Ern, S. Piperno, and K. Djadel. A well- balanced Runge-Kutta discontinuous Galerkin method for the shallow-water equations with flooding and drying. Int. J. Numer. Meth. Fluids, 58:1-25, 2008.

[29] C. Eskilsson and S. J. Sherwin. An hp/spectral element model for efficient long-time integration of Boussinesq-type equations. Coastal Engineering, 45:295 - 320, 2003.

[30] C. Eskilsson and S.J. Sherwin. Discontinuous Galerkin spectral/hp element modelling of dispersive shallow water systems. Journal Of Scientific Computing, 22 - 23:269-288, 2005.

[31] C. Eskilsson and S.J. Sherwin. Spectral/hp discontinuous Galerkin method for modelling 2D Boussinesq equations. J. Comput. Phys., 212:566-589, 2006.

[32] C. Eskilsson, S.J.Sherwin, and L.Bergdahl. An unstructured spectral/hp element model for enhanced Boussinesq-type equations. Coastal Engineering, 53(947-963), 2006. 
[33] S. Gottlieb, C.-W. Shu, and Tadmor E. Strong stability preserving high order time discretization methods. SIAM Review, 43:89-112, 2001.

[34] A.E. Green and P.M. Naghdi. A derivation of equations for wave propagation in water of variable depth. J. Fluid Mech., 78:237-246, 1976.

[35] S. Guibourg. Modélisation numérique et expérimentale des houles bidimensionnelles en zone cotière, phd thesis. Université Joseph Fourier-Grenoble I, France, 1994.

[36] S-C. Hsiao and T-C. Lin. Tsunami-like solitary waves impinging and overtopping an impermeable seawall: Experiment and rans modeling. Coast. Eng., 57:1-18, 2010.

[37] M. Kazolea, A.I. Delis, I.K. Nikolos, and C.E. Synolakis. An unstructured finite volume numerical scheme for extended 2DBoussinesq-type equations. Coast. Eng., 69:42-66, 2012.

[38] M. Kazolea, A.I. Delis, and C.E. Synolakis. Numerical treatment of wave breaking on unstructured finite volume approximations for extended Boussinesq-type equations. J. Comput. Phys., in press, 2014.

[39] A. Kennedy, Q. Chen, J. Kirby, and R. Dalrymple. Boussinesq modeling of wave transformation, breaking, and runup. I: 1 D. Journal of Waterway Port Coastal and Ocean Engineering, 1999.

[40] L. Krivodonova, J. Xin, J.-F. Remacle, N. Chevaugeon, and J.E. Flaherty. Shock detection and limiting with discontinuous Galerkin methods for hyperbolic conservation laws. Applied Numerical Mathematics, 48:323-338, 2004.

[41] D. Lannes. Water waves: mathematical analysis and asymptotics. Amer. Mathematical Society, 2013.

[42] D. Lannes and P. Bonneton. Derivation of asymptotic two-dimensional time-dependent equations for surface water wave propagation. Physics of fluids, 21:016601, 2009.

[43] D. Lannes and F.Marche. A new class of fully nonlinear and weakly dispersive Green-Naghdi models for efficient $2 \mathrm{~d}$ simulations. submitted, 2014.

[44] O. Le Métayer, S. Gavrilyuk, and S. Hank. A numerical scheme for the Green-Naghdi model. J. Comput. Phys., (229):2034$2045,2010$.

[45] M Li, P. Guyenne, F. Li, and F. Xu. High order well-balanced CDG-FE methods for shallow water waves by a Green-Naghdi model. J. Comput. Phys., 257:169-192, 2014.

[46] Y.S. Li, S.X. Liu, Y.X. Yu, and G.Z. Lai. Numerical modeling of Boussinesq equations by finite element method. Coast. Eng., 37:97-122, 1999.

[47] Q. Liang and A. G. L. Borthwick. Adaptive quadtree simulation of shallow flows with wet-dry fronts over complex topography. Computers and Fluids, 38(2):221-234, 2009.

[48] Q. Liang and F. Marche. Numerical resolution of well-balanced shallow water equations with complex source terms. Adv. Water Res., 32:873-884, 2009.

[49] P.A. Madsen, H.B. Bingham, and H. Liu. A new Boussinesq method for fully nonlinear waves from shallow to deep water. J. Fluid Mech., 462:1-30, 2002.

[50] P.A. Madsen, H.B. Bingham, and H.A. Schaffer. Boussinesq-type formulations for fully nonlinear and extremely dispersive water waves: derivation and analysis. Proc. R. Soc. A, 459:1075-1104, 2003.

[51] P.A. Madsen, R. Murray, and O.R. Sorensen. A new form of the Boussinesq equations with improved linear dispersion characteristics. Coastal Engineering, 15(4):371-388, 1991.

[52] P.A. Madsen and O.R. Sørensen. A new form of the Boussinesq equations with improved linear dispersion characteristics. Part 2: A slowing varying bathymetry. Coast. Eng., 18:183-204, 1992.

[53] D. Mitsotakis, B. Ilan, and D. Dutykh. On the Galerkin/finite-element method for the Serre equations. J. Sci. Comput., 2014.

[54] O. Nwogu. Alternative form of Boussinesq equations for nearshore wave propagation. J. Waterway. Port. Coastal. Ocean Eng., 119:618-638, 1993.

[55] J. Orszaghova, A.G.L. Borthwick, and P.H. Taylor. From the paddle to the beach - A Boussinesq shallow water numerical wave tank based on Madsen and Sørensen's equations. J. Comp. Phys., 231:328-344, 2012.

[56] J.D. Pearce and J.G. Esler. A pseudo-spectral algorithm and test cases for the numerical solution of the two-dimensional rotating Green-Naghdi shallow water equations. J. Comput. Phys., 229:7594-7608, 2010.

[57] D. Peregrine. Long waves on a beach. Journal of Fluid Mechanics, 27:815-827, 1967.

[58] J. Qiu and C.-W. Shu. A comparison of troubled-cell indicators for Runge-Kutta discontinuous Galerkin methods using weighted essentially nonoscillatory limiters. SIAM J. Sci. Comput., 27:995-1013, 2005.

[59] M. Ricchiuto and A.G. Filippini. Upwind residual discretization of enhanced Boussinesq equations for wave propagation over complex bathymetries. J. Comput. Phys., in press, 2014.

[60] V. Roeber and K.F. Cheung. Boussinesq-type model for energetic breaking waves in fringing reef environments. Coastal Engineering, 70(0):1 - 20, 2012.

[61] B. Rogers, M. Fujihara, and A. Borthwick. Adaptive Q-tree Godunov-type scheme for shallow water equations. Int. J. Numer. Methods Fluids, 35:247-280, 2001.

[62] F. Serre. Contribution á l'étude des écoulements permanents et variables dans les canaux. Houille Blanche, 6:830-872, 1953.

[63] F. Shi, J.T. Kirby, J.C. Harris, J.D. Geiman, and S.T. Grilli. A high-order adaptive time-stepping tvd solver for Boussinesq modeling of breaking waves and coastal inundation. Ocean Modelling, 43-44:36-51, 2012. 
[64] S. Soares-Frazão and V. Guinot. A second-order semi-implicit hybrid scheme for one-dimensional Boussinesq-type waves in rectangular channels. Int. J. For Num. Meth. Fluids, 58:237-261, 2008.

[65] O. Sørensen, H. Schäffer, and P. Madsen. Surf zone dynamics simulated by a Boussinesq type model. III. Wave-induced horizontal nearshore circulation. Coastal Engineering, 33:155-176, 1998.

[66] O.R. Sørensen, H.A. Schaffer, and L.S. Sørensen. Boussinesq-type modelling using an unstructured finite element technique. Coastal Engineering, 50:181-198, 2004.

[67] C.E. Synolakis. The runup of solitary waves. J. Fluid Mech., 185:523-545, 1981.

[68] M. Tissier, P. Bonneton, F. Marche, F. Chazel, and D. Lannes. A new approach to handle wave breaking in fully non-linear Boussinesq models. Coastal Engineering, 67:54-66, 2012.

[69] M. Tonelli and M. Petti. Hybrid finite volume- finite difference scheme for 2dh improved Boussinesq equations. Coastal Engineering, 56(5-6):609-620, 2009.

[70] M. Tonelli and M. Petti. Finite volume scheme for the solution of $2 \mathrm{~d}$ extended Boussinesq equations in the surf zone. Ocean Engineering, 37:567-582, 2010.

[71] M. Tonelli and M. Petti. Shock-capturing Boussinesq model for irregular wave propagation. Coastal Engineering, 61:8-19, 2012.

[72] E.F. Toro, M. Spruce, and W. Speare. Restoration of the contact surface in the HLL Riemann solver. Shock waves, 4:25-34, 1994.

[73] M. Walkley and M. Berzins. A finite element method for the two-dimensional extended Boussinesq equations. Internat. J. Numer. Methods Fluids, 39(10):865-885, 2002

[74] Y. Wang, Q. Liang, G. Kesserwani, and J.W. Hall. A 2D shallow flow model for practical dam-break simulations. J. Hydraulic Res., 49(3):307-316, 2011.

[75] G. Wei and J. Kirby. Time-dependent numerical code for extended Boussinesq equations. Journal of Waterway, 121:251-261, 1995.

[76] G. Wei, J. Kirby, S. Grilli, and R. Subramanya. A fully nonlinear Boussinesq model for surface waves. Part 1. Highly nonlinear unsteady waves. Journal of Fluid Mechanics, 294:71-92, 1995.

[77] Y. Xing and C.-W. Shu. A new approach of high order well-balanced finite volume WENO schemes and discontinuous Galerkin methods for a class of hyperbolic systems with source terms. Commun. Comput. Phys., 1:100-134, 2006.

[78] Y. Xing and X. Zhang. Positivity-preserving well-balanced discontinuous Galerkin methods for the shallow water equations on unstructured triangular meshes. J. Sci. Comput., 57:19-41, 2013.

[79] Y. Xing, X. Zhang, and C.-W. Shu. Positivity-preserving high order well-balanced discontinuous Galerkin methods for the shallow water equations. Advances in Water Resources, 33(12):1476 - 1493, 2010.

[80] Y. Xu and C.-W. Shu. Local Discontinuous Galerkin methods for high-order time-dependent partial differential equations. Commun. Comput. Phys., 7(1):1-46, 2010.

[81] X. Zhang and C.-W. Shu. On maximum-principle-satisfying high order schemes for scalar conservation laws. J. Comput. Phys., $229(9): 3091-3120,2010$. 